\title{
On Fuzzy Rough Sets and Their Topological Structures
}

\author{
Weidong Tang, ${ }^{1}$ Jinzhao $\mathrm{Wu},{ }^{1,2,3}$ and Dingwei Zheng ${ }^{4}$ \\ ${ }^{1}$ Chengdu Institute of Computer Applications, Chinese Academy of Sciences, Chengdu 610054, China \\ ${ }^{2}$ Guangxi University for Nationalities, Nanning 530006, China \\ ${ }^{3}$ Guangxi Key Laboratory of Hybrid Computational and IC Design Analysis, Nanning 530006, China \\ ${ }^{4}$ College of Mathematics and Information Science, Guangxi University, Nanning 530004, China
}

Correspondence should be addressed to Jinzhao Wu; himrwujz@126.com

Received 30 May 2013; Revised 18 November 2013; Accepted 19 November 2013; Published 27 January 2014

Academic Editor: Jun-Juh Yan

Copyright (C) 2014 Weidong Tang et al. This is an open access article distributed under the Creative Commons Attribution License, which permits unrestricted use, distribution, and reproduction in any medium, provided the original work is properly cited.

The core concepts of rough set theory are information systems and approximation operators of approximation spaces. Approximation operators draw close links between rough set theory and topology. This paper is devoted to the discussion of fuzzy rough sets and their topological structures. Fuzzy rough approximations are further investigated. Fuzzy relations are researched by means of topology or lower and upper sets. Topological structures of fuzzy approximation spaces are given by means of pseudoconstant fuzzy relations. Fuzzy topology satisfying (CC) axiom is investigated. The fact that there exists a one-to-one correspondence between the set of all preorder fuzzy relations and the set of all fuzzy topologies satisfying $(\mathrm{CC})$ axiom is proved, the concept of fuzzy approximating spaces is introduced, and decision conditions that a fuzzy topological space is a fuzzy approximating space are obtained, which illustrates that we can research fuzzy relations or fuzzy approximation spaces by means of topology and vice versa. Moreover, fuzzy pseudoclosure operators are examined.

\section{Introduction}

Rough set theory, proposed by Pawlak [1], is a new mathematical tool for data reasoning. It may be seen as an extension of classical set theory and has been successfully applied to machine learning, intelligent systems, inductive reasoning, pattern recognition, mereology, image processing, signal analysis, knowledge discovery, decision analysis, expert systems, and many other fields [2-5].

The basic structure of rough set theory is an approximation space. Based on it, lower and upper approximations can be induced. Using these approximations, knowledge hidden in information systems may be revealed and expressed in the form of decision rules (see [2-4]). A key notion in Pawlak rough set model is equivalence relations. The equivalence classes are the building blocks for the construction of these approximations. In the real world, the equivalence relation is, however, too restrictive for many practical applications. To address this issue, many interesting and meaningful extensions of Pawlak rough sets have been presented. Equivalence relations can be replaced by tolerance relations [6], similarity relations [7], binary relations [8-10], and so on.

Various fuzzy generalizations of rough approximations have been proposed [11-16]. The most common fuzzy rough set is obtained by replacing the crisp relations with fuzzy relations on the universe and crisp subsets with fuzzy sets. Dubois and Prade [11] first proposed the concept of rough fuzzy sets and fuzzy rough sets and pointed out that a rough fuzzy set is a special case of a fuzzy rough set. Now, fuzzy rough sets have been used to solve practical problems such as data mining [17], approximate reasoning [5], medical time series, and case generation [18].

There are two approaches to the development of fuzzy rough set theory as in classical cases. One is the constructive approach in which lower and upper approximation operators are constructed from fuzzy relations. The other one is the axiomatic approach in which the lower and upper approximation operators satisfy a set of axioms that are the same as those constructed ones. Morsi and Yakout [19] provide the axioms for fuzzy rough set model. Wu et al. [16] presented 
a general framework for the study of fuzzy rough sets in which both constructive and axiomatic approaches are used. Yeung et al. [20] researched the generalization of fuzzy rough sets and established a connection between fuzzy preorders and saturated Kuratowski fuzzy closure operators satisfying an additional condition.

Topological structure is an important base for knowledge extraction and processing. Therefore, an interesting and natural research topic in rough set theory is to study the relationship between rough sets and topologies. Many authors studied topological structures of rough sets [21-26]. It is known that the pair of lower and upper approximation operators induced by a reflexive and transitive relation is exactly the pair of interior and closure operators of a topology $[21,27]$.

In the study of topological structures of fuzzy rough sets or fuzzy approximation spaces, Qin and Pei [28] investigated topological structures of fuzzy rough sets and pointed out that there exists a one-to-one correspondence between the set of all reflexive, transitive fuzzy relations and the set of all fuzzy topologies satisfying (TC) axiom; Hao and Li [29] discussed the relationship between $L$-fuzzy rough set and $L$-topology. Zhou et al. [30] and Wu and Zhou [31] studied intuitionistic fuzzy rough sets and intuitionistic fuzzy topologies.

Topological structures of approximation spaces may have some applications in information science. In practical, it often happens that some attribute values for an object of an information system are missing. For such incomplete information systems, Kryszkiewicz [32, 33] and Salama [34] studied the rule generation and information recovery by rough set approach and topological method, respectively.

The purpose of this paper is to investigate further topological structures of fuzzy approximation spaces.

The remaining part of this paper is organized as follows. In Section 2, we recall some basic concepts about fuzzy sets and fuzzy topologies. In Section 3, fuzzy rough approximation operators are further investigated and fuzzy relations are researched by means of topology or lower and upper sets. In Section 4, topological structures of fuzzy approximation spaces are given. In Section 5, fuzzy topology satisfying (CC) axiom is investigated, concept of fuzzy approximating spaces is introduced, and the decision conditions that a fuzzy topological space is a fuzzy approximating space are obtained. In Section 6, fuzzy pseudoclosure operators are considered. Some concluding remarks appear in Section 7.

\section{Preliminaries}

Throughout this paper, $U$ denotes a nonempty finite set called the universe of discourse. $I$ denotes $[0,1] .2^{U}$ denotes the family of all subsets of $U . F(U)$ denotes the set of all fuzzy sets in $U$. For $a \in I, \bar{a}$ denotes the constant fuzzy set in $U$.

We recall some basic operations on $F(U)$ as follows [35]: for any $A, B \in F(U)$ and $\lambda \in I$,

(1) $A=B \Leftrightarrow A(x)=B(x)$ for each $x \in U$,

(2) $A \subseteq B \Leftrightarrow A(x) \leq B(x)$ for each $x \in U$,

(3) $A=B^{c} \Leftrightarrow A(x)=1-B(x)$ for each $x \in U$,
(4) $(A \cap B)(x)=A(x) \wedge B(x)$ for each $x \in U$,

(5) $(A \cup B)(x)=A(x) \vee B(x)$ for each $x \in U$.

Moreover,

$$
\begin{aligned}
& \left(\bigcup_{j \in J} A\right)(x)=\bigvee_{j \in J} A(x), \\
& \left(\bigcap_{j \in J} A\right)(x)=\bigwedge_{j \in J} A(x),
\end{aligned}
$$

where $\left\{A_{j}: j \in J\right\} \subseteq F(U)$.

(6) $(\lambda A)(x)=\lambda \wedge A(x)$ for each $x \in U$.

For each $A \in F(U)$, we denote

$$
R_{A}=\{(x, y) \in U \times U: A(x)>A(y)\} .
$$

Obviously, $R_{A}=\emptyset \Leftrightarrow A=\bar{a}$ for some $\lambda \in I$.

A fuzzy set is called a fuzzy point in $U$, if it takes the value 0 for each $y \in U$ except one, say, $x \in U$. If its value at $x$ is $\lambda(0<\lambda \leq 1)$, we denote this fuzzy point by $x_{\lambda}$, where the point $x$ is called its support and $\lambda$ is called its height (see [36]). Denote

$$
P(U)=\left\{x_{\lambda}: x \in U, \lambda \in(0,1]\right\} .
$$

Specially,

$$
x_{1}(y)= \begin{cases}1, & y=x, \\ 0, & y \in U-\{x\} .\end{cases}
$$

For a fuzzy point $x_{\lambda}$ and $A \in F(U)$, we defined $x_{\lambda} \in A$ by $x_{\lambda} \subseteq A$.

Obviously,

$$
x_{\lambda} \in A \Longleftrightarrow \lambda \leq A(x) .
$$

Remark 1. Consider $A=\bigcup_{x \in U}\left(A(x) x_{1}\right)$ and $A=\bigcap_{x \in U}(\overline{A(x)}$ $\left.\cup x_{1}\right)(A \in F(U))$.

Definition 2 (see [37]). Consider $\tau \subseteq F(U)$ is called a fuzzy topology on $U$, if

(i) for each $a \in I, \bar{a} \in \tau$;

(ii) $A, B \in \tau$ implies $A \cap B \in \tau$;

(iii) $\left\{A_{j}: j \in J\right\} \subseteq \tau$ implies $\bigcup_{j \in J} A_{j} \in \tau$.

The pair $(U, \tau)$ is called a fuzzy topological space. Every member of $\tau$ is called a fuzzy open set in $U$. Its complement is called a fuzzy closed set in $U$.

We denote $\tau^{c}=\left\{A \in F(U): A^{c} \in \tau\right\}$.

The interior and closure of $A$ denoted, respectively, by $\operatorname{int}(A)$ and $\operatorname{cl}(A)$ for each $A \in F(U)$ are defined as follows:

$$
\begin{aligned}
& \operatorname{int}(A) \text { or } \operatorname{int}_{\tau}(A)=\bigcup\{B \in \tau: B \subseteq A\}, \\
& \operatorname{cl}(A) \text { or } \operatorname{cl}_{\tau}(A)=\bigcap\left\{B \in \tau^{c}: B \supseteq A\right\} .
\end{aligned}
$$


It should be pointed out that if (i) in Definition 2 is replaced by

(i) ${ }^{\prime} \overline{0}, \overline{1} \in \tau$,

then $\tau$ is a fuzzy topology in the sense of Chang [38]. We can see that a fuzzy topology in the sense of Lowen must be a fuzzy topology in the sense of Chang. In this paper, we always consider the fuzzy topology in the sense of Lowen.

A fuzzy topology $\tau$ is called Alexandrov [39] if (ii) in Definition 2 is replaced by

(ii) ${ }^{\prime}\left\{A_{j}: j \in J\right\} \subseteq \tau \Rightarrow \bigcap_{j \in J} A_{j} \in \tau$.

Definition 3 (see [36]). Let $(U, \tau)$ be a fuzzy topological space and let $x_{\lambda} \in P(U)$ and $A \in F(U)$. $A$ is called a closed remote neighborhood of $x_{\lambda}$, if $A \in \tau^{c}$ and $x_{\lambda} \notin A$.

Denote

$$
\eta^{-}\left(x_{\lambda}\right)=\{A \in F(U)
$$

: $A$ is closed remote neighborhood of $\left.x_{\lambda}\right\}$.

Definition 4 (see [36]). Let $(U, \tau)$ be a fuzzy topological space. Consider the following.

(1) $(U, \tau)$ is called $T_{-1}$, if for any $x_{\lambda}, x_{\mu} \in P(U)$ and $\mu<\lambda$, there exists $A \in \eta^{-}\left(x_{\lambda}\right)$ such that $x_{\mu} \in A$, or, there exists $B \in \eta^{-}\left(x_{\mu}\right)$ such that $x_{\lambda} \in B$.

(2) $(U, \tau)$ is sub- $T_{0}$, if for any $x, y \in U$ and $x \neq y$, there exist $\lambda \in(0,1]$ and $A \in \eta^{-}\left(x_{\lambda}\right)$ such that $y_{\lambda} \in A$, or, there exist $\lambda \in(0,1]$ and $B \in \eta^{-}\left(y_{\lambda}\right)$ such that $x_{\lambda} \in B$.

Definition 5 (see [40]). Let $R$ be a relation on $U$. For each $x \in$ $U$, denote

$$
\begin{aligned}
& R_{p}(x)=\{y \in U:(y, x) \in R\}, \\
& R_{s}(x)=\{y \in U:(x, y) \in R\} .
\end{aligned}
$$

$R_{p}(x)$ and $R_{s}(x)$ are called the predecessor and successor neighborhood of $x$, respectively.

\section{Fuzzy Rough Sets and Fuzzy Rough Approximation Operators}

In this section, we review rough approximation operators and rough sets in fuzzy environments and further researched their properties.

Recall that $R$ is a fuzzy relation on $U$ if $R \in F(U \times U)$. $R$ may be represented by a matrix. That is, if $U=\left\{t_{1}, t_{2}, \ldots, t_{n}\right\}$, then $R$ may be represented by the following matrix:

$$
\left(\begin{array}{cccc}
R\left(t_{1}, t_{1}\right) & R\left(t_{1}, t_{2}\right) & \cdots & R\left(t_{1}, t_{n}\right) \\
R\left(t_{2}, t_{1}\right) & R\left(t_{2}, t_{2}\right) & \cdots & R\left(t_{2}, t_{n}\right) \\
\cdots & \cdots & \cdots & \cdots \\
R\left(t_{n}, t_{1}\right) & R\left(t_{n}, t_{2}\right) & \cdots & R\left(t_{n}, t_{n}\right)
\end{array}\right) .
$$

Let $R$ be a fuzzy relation on $U . R$ is called reflexive if $R(x, x)=1$ for each $x \in U . R$ is called symmetric if $R(x, y)=R(y, x)$ for any $x, y \in U . R$ is called transitive if $R(x, z) \geq R(x, y) \wedge R(y, z)$ for any $x, y, z \in U . R$ is called preorder if $R$ is reflexive and transitive.

Let $R$ be a fuzzy relation on $U . R^{-1}$ is called the inverse of $R$ if $R^{-1}(x, y)=R(y, x)$ for any $x, y \in U$. For each $z \in U$, we define fuzzy sets $[z]^{R}: U \rightarrow I,[z]^{R}(x)=R(z, x)$, and $[z]_{R}: U \rightarrow I,[z]_{R}(x)=R(x, z)$.

Definition 6 (see [28]). Let $R$ be a fuzzy relation on $U$. The pair $(U, R)$ is called a fuzzy approximation space. The fuzzy lower and the fuzzy upper approximation of $A \in F(U)$ with respect to $(U, R)$, denoted by $\underline{R}(A)$ and $\bar{R}(A)$, are respectively, defined as follows:

$$
\begin{gathered}
\underline{R}(A)(x)=\bigwedge_{y \in U}(A(y) \vee(1-R(x, y))) \quad(x \in U), \\
\bar{R}(A)(x)=\bigvee_{y \in U}(A(y) \wedge R(x, y)) \quad(x \in U) .
\end{gathered}
$$

The pair $(\underline{R}(A), \bar{R}(A))$ is called the fuzzy rough set of $A$ with respect to $(U, R)$.

$\underline{R}: F(U) \rightarrow F(U)$ and $\bar{R}: F(U) \rightarrow F(U)$ are called the fuzzy lower approximation operator and the fuzzy upper approximation operator, respectively. In general, we refer to $\underline{R}$ and $\bar{R}$ as the fuzzy rough approximation operators.

Remark 7. Let $(U, R)$ be a fuzzy approximation space. Then

$$
\begin{gathered}
\bar{R}\left(x_{1}\right)(y)=R(y, x), \\
\underline{R}\left(\left(x_{1}\right)^{c}\right)(y)=1-R(y, x) \quad(x, y \in U) .
\end{gathered}
$$

Let $(U, R)$ be a fuzzy approximation space. $(U, R)$ is call reflexive (resp., preorder) if $R$ is reflexive (resp., preorder).

Proposition 8 (see $[15,28])$. Let $(U, R)$ be a fuzzy approximation space. Then for any $A, B \in F(U),\left\{A_{j}: j \in J\right\} \subseteq F(U)$ and $\lambda \in I$,

(1) $\underline{R}(\overline{1})=\overline{1}, \bar{R}(\overline{0})=\overline{0}$,

(2) $A \subseteq B \Rightarrow \underline{R}(A) \subseteq \underline{R}(B), \bar{R}(A) \subseteq \bar{R}(B)$,

(3) $\underline{R}\left(A^{c}\right)=(\bar{R}(A))^{c}, \bar{R}\left(A^{c}\right)=(\underline{R}(A))^{c}$,

(4) $\underline{R}(A \cap B)=\underline{R}(A) \cap \underline{R}(B), \bar{R}(A \cup B)=\bar{R}(A) \cup \bar{R}(B)$,

(5) $\underline{R}\left(\bigcap_{j \in J} A_{j}\right)=\bigcap_{j \in J}\left(\underline{R}\left(A_{j}\right)\right), \bar{R}\left(\bigcup_{j \in J} A_{j}\right)=$ $\bigcup_{j \in J}\left(\bar{R}\left(A_{j}\right)\right)$.

(6) $\underline{R}(\bar{\lambda} \cup A)=\bar{\lambda} \cup \underline{R}(A), \bar{R}(\lambda A)=\lambda \bar{R}(A)$. 
Theorem 9 (see $[16,28,41])$. Let $(U, R)$ be a fuzzy approximation space. Then Consider the following

(1) $R$ is reflexive

$$
\begin{array}{ll}
\Longleftrightarrow(I L R) & \forall A \in F(U), \underline{R}(A) \subseteq A \\
\Longleftrightarrow(I U R) \quad \forall A \in F(U), A \subseteq \bar{R}(A) .
\end{array}
$$

(2) $R$ is symmetric

$$
\begin{aligned}
& \Longleftrightarrow(I L S) \quad \forall(x, y) \in U \times U, \underline{R}\left(\left(x_{1}\right)^{c}\right)(y) \\
& =\underline{R}\left(\left(y_{1}\right)^{c}\right)(x) \\
& \Longleftrightarrow(I U S) \quad \forall(x, y) \in U \times U, \bar{R}\left(x_{1}\right)(y) \\
& =\bar{R}\left(y_{1}\right)(x) .
\end{aligned}
$$

(3) $R$ is transitive

$$
\begin{array}{ll}
\Longleftrightarrow(I L T) & \forall A \in F(U), \underline{R}(A) \subseteq \underline{R}(\underline{R}(A)) \\
\Longleftrightarrow(I U T) & \forall A \in F(U), \bar{R}(\bar{R}(A)) \subseteq \bar{R}(A) .
\end{array}
$$

Theorem 10 (see $[16,28])$. Let $(U, R)$ be a preorder fuzzy approximation space. Then for each $A \in F(U)$,

$$
\begin{aligned}
& \underline{R}(\underline{R}(A))=\underline{R}(A), \\
& \bar{R}(\bar{R}(A))=\bar{R}(A) .
\end{aligned}
$$

Remark 11. (1) For each $a \in I, \bar{R}(\bar{a}) \subseteq \bar{a} \subseteq \underline{R}(\bar{a})$.

(2) If $R$ is reflexive, then for each $a \in I, R(\bar{a})=\bar{a}=\bar{R}(\bar{a})$.

Definition 12. Let $(U, R)$ be a fuzzy approximation space.

(1) $A \in F(U)$ is called an upper set if $A(x) \bigwedge R(x, y) \leq$ $A(y)$ for any $x, y \in U$.

(2) $A \in F(U)$ is called a lower set if $A(y) \wedge R(x, y) \leq$ $A(x)$ for any $x, y \in U$.

Proposition 13. Let $(U, R)$ be a fuzzy approximation space. Then the following are equivalent:

(1) $\bar{R}(A) \subseteq A$;

(2) $A$ is a lower set in $(U, R)$;

(3) $A$ is an upper set in $\left(U, R^{-1}\right)$.

Proof. $(1) \Rightarrow(2)$. Suppose that $\bar{R}(A) \subseteq A$. Since for each $x \in U$,

$$
\bigvee_{y \in U}(A(y) \wedge R(x, y))=\bar{R}(A)(x) \leq A(x),
$$

we have

$$
A(y) \wedge R(x, y) \leq A(x) \quad(x, y \in U) .
$$

Thus $A$ is a lower set in $(U, R)$.

$(2) \Rightarrow(3)$. This is obvious.
(3) $\Rightarrow(1)$. Suppose that $A$ is an upper set in $\left(X, R^{-1}\right)$. Then for any $x, y \in U, A(x) \wedge R^{-1}(x, y) \leq A(y)$. So $A(x) \wedge R(y, x) \leq$ $A(y)$. Thus

$$
\bar{R}(A)(y)=\bigvee_{x \in U}(A(x) \wedge R(y, x)) \leq A(y) \quad(y \in U) .
$$

Hence $\bar{R}(A) \subseteq A$.

Corollary 14. Let $(U, R)$ be a fuzzy approximation space. If $R$ is reflexive, then the following are equivalent:

(1) $\bar{R}(A)=A$;

(2) $A$ is a lower set in $(U, R)$;

(3) $A$ is an upper set in $\left(U, R^{-1}\right)$.

Proof. This holds by Theorem 9(1) and Proposition 13.

Theorem 15. Let $(U, R)$ be a fuzzy approximation space. Then

(1) $R$ is reflexive

$$
\begin{aligned}
& \Longleftrightarrow\left(I L S^{\prime}\right) \quad \forall x \in U,[x]_{R}(x)=1 \\
& \Longleftrightarrow\left(I U R^{\prime}\right) \quad \forall x \in U,[x]^{R}(x)=1 .
\end{aligned}
$$

(2) $R$ is symmetric

$$
\Longleftrightarrow\left(I L S^{\prime}\right) \quad \forall x \in U,[x]_{R}=[x]^{R}
$$

$\Longrightarrow \forall A \in F(U), \quad A$ is a lower set if and only if $A$ is an upper set.

(3) $R$ is transitive

$\Longleftrightarrow\left(I L T^{\prime}\right) \quad \forall x \in U,[x]_{R}$ is a lower set $\Longleftrightarrow\left(I U T^{\prime}\right) \quad \forall x \in U,[x]^{R}$ is an upper set $\Longleftrightarrow\left(I U T^{\prime \prime}\right) \quad \forall A \in F(U), \bar{R}(A)$ is a lower set.

Proof. (1) and (2) are obvious.

(3)

$\left(I U T^{\prime \prime}\right)$

$\Longleftrightarrow \forall A \in F(U), \quad \bar{R}(\bar{R}(A)) \subseteq \bar{R}(A)$. (Proposition 13)

$\Longleftrightarrow R$ is transitive. (Theorem 9(3))

$\Longleftrightarrow \forall x, y, z \in U, \quad R(x, y) \wedge R(y, z) \leq R(x, z)$

$\Longleftrightarrow \forall x, y, z \in U, \quad R(x, y) \wedge[z]_{R}(y) \leq[z]_{R}(x)$

$\Longleftrightarrow\left(I L T^{\prime}\right) \forall x \in U, \quad[x]_{R}$ is a lower set 


$$
\begin{aligned}
& \Longleftrightarrow \forall x, y, z \in U, \quad[x]^{R}(y) \wedge R(y, z) \leq[x]^{R}(z) \\
& \Longleftrightarrow\left(I U T^{\prime}\right) \forall x \in U, \quad[x]^{R} \text { is an upper set. }
\end{aligned}
$$

Theorem 16 below illustrates that fuzzy relations can be researched by means of topology.

Theorem 16. Let $R$ be a fuzzy relation on $U$ and let $\tau$ be a fuzzy topology on $U$. If one of the following conditions is satisfied, then $R$ is preorder:

(1) $\bar{R}$ is the closure operator of $\tau$;

(2) $\underline{R}$ is the interior operator of $\tau$.

Proof. (1) By Remark 7, $\bar{R}\left(x_{1}\right)(y)=R(y, x)$ for each $x \in U$. Note that $\underline{R}$ is the interior operator of $\tau$. Then for each $x \in U$,

$$
R(x, x)=\bar{R}\left(x_{1}\right)(x)=\mathrm{cl}_{\tau}\left(x_{1}\right)(x) \geq x_{1}(x)=1 .
$$

Thus $R$ is reflexive.

For any $x, y, z \in U$, denote $\mathrm{cl}_{\tau}\left(z_{1}\right)(y)=\lambda$, and by Remarks 1 and 7 and Proposition 8(6),

$$
\begin{aligned}
R(x, y) & \wedge R(y, z) \\
= & \bar{R}\left(y_{1}\right)(x) \wedge \bar{R}\left(z_{1}\right)(y) \\
= & \bar{R}\left(y_{1}\right)(x) \wedge \mathrm{cl}_{\tau}\left(z_{1}\right)(y) \\
= & \bar{R}\left(y_{1}\right)(x) \wedge \lambda \\
= & \lambda \bar{R}\left(y_{1}\right)(x)=\bar{R}\left(\lambda y_{1}\right)(x) \\
& =\mathrm{cl}_{\tau}\left(\lambda y_{1}\right)(x) \\
& =\mathrm{cl}_{\tau}\left(\mathrm{cl}_{\tau}\left(z_{1}\right)(y) y_{1}\right)(x) \\
& \leq \mathrm{cl}_{\tau}\left(\bigcup_{t \in U}\left(\mathrm{cl}_{\tau}\left(z_{1}\right)(t) t_{1}\right)\right)(x) \\
& =\mathrm{cl}_{\tau}\left(\mathrm{cl}_{\tau}\left(z_{1}\right)\right)(x) \\
& =\mathrm{cl}_{\tau}\left(z_{1}\right)(x)=R(x, z) .
\end{aligned}
$$

Then $R$ is transitive. Thus $R$ is preorder.

(2) The proof is similar to (1).

Proposition 17. Let $(U, R)$ be a fuzzy approximation space. Then for each $A \in F(U)$ with $R_{A} \neq \emptyset$, Consider the following.

(1) (a) $\underline{R}(A) \supseteq A \Leftrightarrow(F L O) \forall(x, y) \in R_{A}, 1-R(x, y) \geq$ $\bar{A}(x) \vee A(y)$.

(b) $\bar{R}(A) \subseteq A \Leftrightarrow(F U O) \forall(x, y) \in R_{A}, R(y, x) \leq$ $A(x) \wedge A(y)$.

(2) If $R$ is reflexive, then

(a) $\underline{R}(A)=A \Leftrightarrow(F L R) \forall(x, y) \in R_{A}, 1-R(x, y) \geq$ $A(x) \vee A(y)$.

(b) $\bar{R}(A)=A \Leftrightarrow(F U R) \forall(x, y) \in R_{A}, R(y, x) \leq$ $A(x) \wedge A(y)$.
Proof

(1) (a) Necessity. Suppose that $\underline{R}(A) \supseteq$ A. Note that for each $x \in U$,

$$
\bigwedge_{y \in U}(A(y) \vee(1-R(x, y)))=(\underline{R}(A))(y) \geq A(x) .
$$

Then $A(y) \vee(1-R(x, y)) \geq A(x)$ for any $x, y \in U$. Since $A(x)>A(y)$ for each $(x, y) \in R_{A}$, we have

$$
1-R(x, y) \geq A(x)=A(x) \vee A(y) \quad\left((x, y) \in R_{A}\right) .
$$

Sufficiency. Suppose that (FLO) holds. Let $x \in U$. Consider the following.

(i) If $y \in\left(R_{A}\right)_{s}(x)$, then

$A(y) \vee(1-R(x, y)) \geq A(y) \vee(A(x) \vee A(y)) \geq A(x)$.

(ii) If $y \notin\left(R_{A}\right)_{s}(x)$, then $A(y) \geq A(x)$ and so

$$
A(y) \vee(1-R(x, y)) \geq A(y) \geq A(x) .
$$

Hence $\underline{R}(A)(x)=\bigwedge_{y \in U}(A(y) \vee(1-R(x, y))) \geq A(x)$.

Thus $\underline{R}(A) \supseteq A$.

(b) Necessity. Suppose that $\bar{R}(A) \subseteq A$. Note that for each $y \in$ U,

$$
\bigvee_{x \in U}(A(x) \wedge R(y, x))=\bar{R}(A)(y) \leq A(y) .
$$

Then $A(x) \wedge R(y, x) \leq A(y)(x, y \in U)$ for any $x, y \in U$. Since $A(x)>A(y)$ for each $(x, y) \in R_{A}$, we have

$$
R(y, x) \leq A(y)=A(x) \wedge A(y)\left((x, y) \in R_{A}\right) .
$$

Sufficiency. Suppose that (FLO) holds. Let $y \in U$.

(i) If $x \in\left(R_{A}\right)_{p}(y)$, then $(x, y) \in R_{A}$ and so

$A(x) \vee R(y, x) \leq A(x) \wedge(A(x) \wedge A(y)) \leq A(y)$.

(ii) If $x \notin\left(R_{A}\right)_{p}(y)$, then $A(x) \leq A(y)$ and so

$$
A(x) \wedge R(y, x) \leq A(x) \leq A(y) .
$$

Thus $(\bar{R}(A))(y)=\bigvee_{x \in U}(A(x) \wedge R(y, x)) \leq A(y)$. Hence $\bar{R}(A) \subseteq A$.

(2) This holds by (1), the reflexivity of $R$ and Theorem 9(1).

Proposition 18. Let $\left(U, R_{1}\right)$ and $\left(U, R_{2}\right)$ be two fuzzy approximation spaces. Then for each $A \in F(U)$.

(1) (a) $R_{1} \subseteq R_{2} \Rightarrow \underline{R_{1}}(A) \supseteq \underline{R_{2}}(A), \overline{R_{1}}(A) \subseteq \overline{R_{2}}(A)$.

(b) $\left(\underline{R_{1} \cup R_{2}}\right)(A)=\underline{R_{1}}(A) \cap \underline{R_{2}}(A),\left(\overline{R_{1} \cup R_{2}}\right)(A)=$ $\overline{R_{1}}(A) \cup \overline{R_{2}}(A)$ 
(2) If $R_{1}$ and $R_{2}$ are reflexive, then

(a) $\underline{R_{2}}\left(\underline{R_{1}}(A)\right) \subseteq\left(\underline{R_{1} \cup R_{2}}\right)(A), \underline{R_{1}}\left(\underline{R_{2}}(A)\right) \subseteq$ $\left.\overline{(R}_{1} \bar{\cup} R_{2}\right)(A)$.

(b) $\overline{R_{2}}\left(\overline{R_{1}}(A)\right) \supseteq\left(\overline{R_{1} \cup R_{2}}\right)(A), \overline{R_{1}}\left(\overline{R_{2}}(A)\right) \supseteq$ $\left(\overline{R_{1} \cup R_{2}}\right)(A)$.

(3) If $R_{1}$ is reflexive, $R_{2}$ is transitive and $R_{1} \subseteq R_{2}$, then

$\underline{R_{1}}\left(\underline{R_{2}}(A)\right)=\underline{R_{2}}(A), \quad \overline{R_{1}}\left(\overline{R_{2}}(A)\right)=\overline{R_{2}}(A)$.

Proof. (1) (a) This is obvious.

(b) For each $x \in U$, since

$$
\begin{aligned}
\left(\overline{R_{1} \cup R_{2}}\right)(A)(x) \\
=\bigvee_{y \in U}\left(A(y) \wedge\left(R_{1} \cup R_{2}\right)(x, y)\right) \\
=\bigvee_{y \in U}\left(A(y) \wedge\left(R_{1}(x, y) \vee R_{2}(x, y)\right)\right) \\
=\bigvee_{y \in U}\left(\left(A(y) \wedge R_{1}(x, y)\right) \vee\left(A(y) \wedge R_{2}(x, y)\right)\right) \\
=\left(\bigvee_{y \in U}\left(A(y) \wedge R_{1}(x, y)\right)\right) \\
\quad \vee\left(\bigvee_{y \in U}\left(A(y) \vee R_{2}(x, y)\right)\right) \\
=\overline{R_{1}}(A)(x) \vee \overline{R_{2}}(A)(x) \\
=\left(\overline{R_{1}}(A) \cup \overline{R_{2}}(A)\right)(x),
\end{aligned}
$$

we have

$$
\left(\overline{R_{1} \cup R_{2}}\right)(A)=\overline{R_{1}}(A) \cup \overline{R_{2}}(A) .
$$

By Proposition 8(3),

$$
\begin{aligned}
\underline{R_{1}}(A) & \cap \underline{R_{2}}(A) \\
= & \left(\overline{R_{1}}\left(A^{c}\right)\right)^{c} \cap\left(\overline{R_{2}}\left(A^{c}\right)\right)^{c} \\
= & \left(\overline{R_{1}}\left(A^{c}\right) \cup \overline{R_{2}}\left(A^{c}\right)\right)^{c} \\
= & \left(\left(\overline{R_{1} \cup R_{2}}\right)\left(A^{c}\right)\right)^{c}=\left(\underline{R_{1} \cup R_{2}}\right)(A) .
\end{aligned}
$$

(2) (a) By Theorem 9(1), $\underline{R_{2}}\left(\underline{R_{1}}(A)\right) \subseteq \underline{R_{2}}(A)$ and $\underline{R_{2}}\left(\underline{R_{1}}(A)\right) \subseteq \underline{R_{1}}(A)$. By $(1)$,

$$
\underline{R_{2}}\left(\underline{R_{1}}(A)\right) \subseteq \underline{R_{1}}(A) \cap \underline{R_{2}}(A)=\left(\underline{R_{1} \cup R_{2}}\right)(A) .
$$

Similarly, we can prove that $R_{1}\left(R_{2}(A)\right) \subseteq\left(R_{1} \cup R_{2}\right)(A)$.

(b) The proof is similar to (a).

(3) By Theorem 9(1), $\overline{R_{1}}\left(\overline{R_{2}}(A)\right) \supseteq \overline{R_{2}}(A)$.
For each $x \in U$, by $R_{1} \subseteq R_{2}$ and the transitivity of $R_{2}$, we have

$$
\begin{aligned}
\overline{R_{1}}\left(\overline{R_{2}}(A)\right)(x) \\
=\bigvee_{y \in U}\left(\overline{R_{2}}(A)(y) \wedge R_{1}(x, y)\right) \\
=\bigvee_{y \in U}\left(\left(\bigvee_{z \in U}\left(A(z) \wedge R_{2}(y, z)\right)\right) \wedge R_{1}(x, y)\right) \\
=\bigvee_{y \in U}\left(\bigvee_{z \in U}\left(\left(A(z) \wedge R_{2}(y, z)\right) \wedge R_{1}(x, y)\right)\right) \\
=\bigvee_{y \in U}\left(\bigvee_{z \in U}\left(A(z) \wedge\left(R_{2}(y, z) \wedge R_{1}(x, y)\right)\right)\right) \\
\leq \bigvee_{y \in U}\left(\bigvee_{z \in U}\left(A(z) \wedge\left(R_{2}(y, z) \wedge R_{2}(x, y)\right)\right)\right) \\
\leq \bigvee_{y \in U}\left(\bigvee_{z \in U}\left(A(z) \wedge R_{2}(x, z)\right)\right) \\
=\bigvee_{z \in U}\left(A(z) \wedge R_{2}(x, z)\right) \\
=\overline{R_{2}}(A)(x) .
\end{aligned}
$$

Then $\overline{R_{1}}\left(\overline{R_{2}}(A)\right) \subseteq \overline{R_{2}}(A)$. So $\overline{R_{1}}\left(\overline{R_{2}}(A)\right)=\overline{R_{2}}(A)$.

By Proposition 8(3),

$$
\begin{aligned}
\underline{R_{1}}\left(\underline{R_{2}}(A)\right) & =\underline{R_{1}}\left(\left(\overline{R_{2}}\left(A^{c}\right)\right)^{c}\right) \\
& =\left(\overline{R_{1}}\left(\overline{R_{2}}\left(A^{c}\right)\right)\right)^{c}=\left(\overline{R_{2}}\left(A^{c}\right)\right)^{c} \\
& =\underline{R_{2}}(A) .
\end{aligned}
$$

\section{Fuzzy Approximation Spaces versus Fuzzy Topologies}

In this section we give topological structures of fuzzy approximation spaces (i.e., structures of the topology induced by fuzzy approximation spaces).

For each $R \in F(U \times U)$, denote

$$
\begin{gathered}
\tau_{R}=\{A \in F(U): A=\underline{R}(A)\}, \\
\theta_{R}=\{\underline{R}(A): A \in F(U)\} ; \\
s_{R}=\bigwedge_{x, y \in U, x \neq y} R(x, y), \\
t_{R}=\bigvee_{x, y \in U, x \neq y} R(x, y) .
\end{gathered}
$$


Proposition 19. Let $(U, R)$ be a fuzzy approximation space. If $R$ is preorder, then

$$
\tau_{R}=\theta_{R}
$$

Proof. Obviously, $\tau_{R} \subseteq \theta_{R}$. By Theorem 10, $\tau_{R} \supseteq \theta_{R}$. Thus $\tau_{R}=\theta_{R}$.

\subsection{The Fuzzy Topology Induced by a Reflexive Fuzzy Approximation Space}

Theorem 20. Let $(U, R)$ be a reflexive fuzzy approximation space. Then Consider the following.

(1) $\tau_{R}$ is an Alexandrov fuzzy topology on $U$.

(2) For each $A \in F(U)$,

$$
\operatorname{int}_{\tau_{R}}(A) \subseteq \underline{R}(A) \subseteq A \subseteq \bar{R}(A) \subseteq \mathrm{cl}_{\tau_{R}}(A) .
$$

(3) $A \in\left(\tau_{R}\right)^{c} \Leftrightarrow A=\bar{R}(A)$.

(4) For each $a \in I, \bar{a} \in\left(\tau_{R}\right)^{c}$.

(5) $\tau_{R}=\left\{A^{c}: A\right.$ is a lower set in $\left.(U, R)\right\}, \tau_{R^{-1}}=\left\{A^{c}\right.$ : $A$ is an upper set in $(U, R)\}$.

Proof. (1) (i) For each $a \in I$, by the reflexivity of $R$ and Remark 11(2), $\underline{R}(\bar{a})=\bar{a}$. Then $\bar{a} \in \tau_{R}$.

(ii) Let $\left\{A_{j}: j \in J\right\} \subseteq \tau_{R}$. Then $\underline{R}\left(A_{j}\right)=A_{j}$ for each $j \in J$. By Proposition 8(5),

$$
\underline{R}\left(\bigcap_{j \in J} A_{j}\right)=\bigcap_{j \in J} \underline{R}\left(A_{j}\right)=\bigcap_{j \in J} A_{j} .
$$

Hence $\bigcap_{j \in J} A_{j} \in \tau_{R}$.

(iii) Let $\left\{A_{j}: j \in J\right\} \subseteq \tau_{R}$. Then $\underline{R}\left(A_{j}\right)=A_{j}$ for each $j \in J$. By the reflexivity of $R$ and Theorem $9(1), \underline{R}\left(\bigcup_{j \in J} A_{j}\right) \subseteq$ $\bigcup_{j \in J} A_{j}$. By Proposition 8(2),

$$
\underline{R}\left(\bigcup_{j \in J} A_{j}\right) \supseteq \bigcup_{j \in J} \underline{R}\left(A_{j}\right)=\bigcup_{j \in J} A_{j} .
$$

Then $\underline{R}\left(\bigcup_{j \in J} A_{j}\right)=\bigcup_{j \in J} A_{j}$. So $\bigcup_{j \in J} A_{j} \in \tau_{R}$.

Hence $\tau_{R}$ is Alexandrov.

(2) For each $A \in F(U)$, by Proposition $8(2)$,

$$
\begin{aligned}
\operatorname{int}_{\tau_{R}} & (A) \\
& =\bigcup\left\{B \in \tau_{R}: B \subseteq A\right\} \\
& \subseteq \bigcup\left\{B \in \tau_{R}: \underline{R}(B) \subseteq \underline{R}(A)\right\} \\
& =\bigcup\{B \in F(U): B=\underline{R}(B) \subseteq \underline{R}(A)\} \\
& \subseteq \underline{R}(A) .
\end{aligned}
$$

By Proposition 8(3),

$$
\mathrm{cl}_{\tau_{R}}(A)=\left(\operatorname{int}_{\tau_{R}}\left(A^{c}\right)\right)^{c} \supseteq\left(\underline{R}\left(A^{c}\right)\right)^{c}=\bar{R}(A) .
$$

By the reflexivity of $R$ and Theorem 9(1),

$$
\operatorname{int}_{\tau_{R}}(A) \subseteq \underline{R}(A) \subseteq A \subseteq \bar{R}(A) \subseteq \mathrm{cl}_{\tau_{R}}(A) .
$$

(3) This holds by Proposition 8(3).

(4) This holds by (3) and Remark 11(2).

(5) This holds by Proposition 8(3) and Corollary 14.

Example 21. Let $U=\{x, y, z, w\}$ and let $R$ be a fuzzy relation on $U$, where

$$
R=\left(\begin{array}{llll}
0 & 1 & 1 & 1 \\
1 & 0 & 1 & 1 \\
1 & 1 & 0 & 1 \\
1 & 1 & 1 & 0
\end{array}\right)
$$

Then $R$ is not reflexive.

For any $A \in F(U)$ and $t \in U$,

$$
\underline{R}(A)(t)=\bigwedge_{s \in U}(A(s) \vee(1-R(t, s)))=\bigwedge_{s \in U-\{t\}} A(s) .
$$

Suppose that $A(x) \leq A(y) \leq A(z) \leq A(w)$. Since $\underline{R}(A)=$ $A$, we have

$$
A(x) \wedge A(y) \wedge A(z)=A(w)
$$

Then $A(t) \geq A(w)$ for each $t \in\{x, y, z\}$. So $A(x)=A(y)=$ $A(z)=A(w)$. Thus $\tau_{R}=\{\bar{a}: a \in I\}$.

Obviously, $\tau_{R}$ is an Alexandrov fuzzy topology on $U$.

Definition 22. Let $(U, R)$ be a reflexive fuzzy approximation space. $\tau_{R}$ is called the fuzzy topology induced by $(U, R)$ or induced by $R$ on $U$.

Theorem 23. Let $(U, R)$ be a reflexive fuzzy approximation space and let $\tau_{R}$ be the fuzzy topology induced by $(U, R)$. Then the following properties hold.

(1) $\left(U, \tau_{R}\right)$ is not connected.

(2) $\left(U, \tau_{R}\right)$ is $T_{-1}$.

(3) (a) If $\left(U, \tau_{R}\right)$ is sub-T, then for any $x, y \in U$ with $x \neq y, R(x, y) \wedge R(y, x)<1$.

(b) If $t_{R}<1$, then $\left(U, \tau_{R}\right)$ is sub- $T_{0}$.

Proof. (1) This holds by Theorem 20.

(2) For any $x_{\lambda}, x_{\mu} \in P(U)$ with $\mu<\lambda$, put $u \in(\mu, \lambda)$ and we have $x_{\lambda} \notin \bar{u}$. By Theorem 20(4), $\bar{u} \in \eta^{-}\left(x_{\lambda}\right)$. Note that $x_{\mu} \in \bar{u}$. Then $\left(U, \tau_{R}\right)$ is $T_{-1}$.

(3) (a) For any $x, y \in U$ with $x \neq y$, since $\left(U, \tau_{R}\right)$ is sub- $T_{0}$, then there exist $\lambda \in(0,1]$ and $A \in \eta^{-}\left(x_{\lambda}\right)$ such that $y_{\lambda} \in A$, or there exist $\lambda \in(0,1]$ and $B \in \eta^{-}\left(y_{\lambda}\right)$ such that $x_{\lambda} \in B$.

(i) If there exist $\lambda \in(0,1]$ and $A \in \eta^{-}\left(x_{\lambda}\right)$ such that $y_{\lambda} \in$ $A$, then $A(y) \geq \lambda>A(x)$. By Theorem 20(3) and $A \in$ $\left(\tau_{R}\right)^{c}, \bar{R}(A)=A$. By $(y, x) \in R_{A}$ and Proposition 17(2),

$$
R(x, y) \leq A(x) \wedge A(y)=A(x)<\lambda \leq 1 .
$$

Then $R(x, y)<1$. 
(ii) If there exist $\lambda \in(0,1]$ and $B \in \eta^{-}\left(y_{\lambda}\right)$ such that $x_{\lambda} \in$ $B$, then we can prove that $R(y, x)<1$.

So $R(x, y) \wedge R(y, x)<1$.

(b) Let $x, y \in U$ with $x \neq y$. By $t_{R}<1$, there exists $\lambda \in$ $\left(t_{R}, 1\right]$. Put

$$
A(t)= \begin{cases}t_{R}, & t=x \\ \lambda, & t \neq x\end{cases}
$$

Then $R_{A}=\{(t, x): t \in U-\{x\}\}$. For each $(t, x) \in R_{A}$,

$$
\begin{aligned}
R(x, t) & \leq \bigvee_{x, y \in U, x \neq y} R(x, y)=t_{R} \\
& =\lambda \wedge t_{R}=A(t) \wedge A(x) .
\end{aligned}
$$

By Proposition 17(2), $\bar{R}(A)=A$. By Theorem 20(3), $A \in\left(\tau_{R}\right)^{c}$. Note that $A(x)=t_{R}<\lambda$. Then $A \in \eta^{-}\left(x_{\lambda}\right)$. Obviously, $y_{\lambda} \in$ $A$. Hence $\left(U, \tau_{R}\right)$ is sub $-T_{0}$.

Proposition 24. Let $R_{1}$ and $R_{2}$ be two reflexive fuzzy relations on $U$. Then

(1) $R_{1} \subseteq R_{2} \Rightarrow \tau_{R_{2}} \subseteq \tau_{R_{1}}$.

(2)

$$
\begin{aligned}
\tau_{R_{1}} & \subseteq \tau_{R_{2}} \Longrightarrow \bigvee_{y \in U-\{x\}} R_{2}(x, y) \\
& \leq \bigvee_{y \in U-\{x\}} R_{1}(x, y) \quad(x \in U) .
\end{aligned}
$$

(3) $R_{1} \cup R_{2}$ is reflexive.

(4) $\tau_{R_{1} \cup R_{2}}$ is an Alexandrov fuzzy topology on $U$.

(5) $\tau_{R_{1} \cup R_{2}}=\tau_{R_{1}} \cap \tau_{R_{2}}$.

Proof. (1) For each $A \in \tau_{R_{2}}, R_{2}(A)=A$. By $R_{1} \subseteq R_{2}$, Proposition 18(1), the reflexivity of $R_{1}$ and Theorem 9(1),

$$
A=\underline{R_{2}}(A) \subseteq \underline{R_{1}}(A) \subseteq A .
$$

Hence $A \in \tau_{R_{1}}$. Thus $\tau_{R_{2}} \subseteq \tau_{R_{1}}$.

(2) Let $x \in U$. Pick $\lambda=1-\bigvee_{y \in U-\{x\}} R_{1}(x, y)$.

(i) If $\lambda=0$, then

$$
\bigvee_{y \in U-\{x\}} R_{2}(x, y) \leq 1=\bigvee_{y \in U-\{x\}} R_{1}(x, y)
$$

(ii) If $\lambda>0$, put $A=x_{\lambda} \in P(U)$, then $R_{A}=\{(x, y): y \in$ $U-\{x\}\}$. For any $(x, y) \in R_{A}$, by

$$
1-R_{1}(x, y) \geq 1-\bigvee_{y \in U-\{x\}} R_{1}(x, y)=\lambda=A(x) \vee A(y)
$$

and Proposition 17(2), $R_{1}(A)=A$. So $A \in \tau_{1}$. Note that $\tau_{R_{1}} \subseteq$ $\tau_{R_{2}}$. Then $A \in \tau_{2}$. So $\overline{R_{2}}(A)=A$. For each $(x, y) \in R_{A}$, by Proposition 17(2),

$$
1-R_{2}(x, y) \geq A(x) \vee A(y)=\lambda .
$$

Hence

$$
\begin{aligned}
1- & \bigvee_{y \in U-\{x\}} R_{2}(x, y) \\
& =\bigwedge_{y \in U-\{x\}}\left(1-R_{2}(x, y)\right) \geq \lambda \\
& =1-\bigvee_{y \in U-\{x\}} R_{1}(x, y) .
\end{aligned}
$$

So $\bigvee_{y \in U-\{x\}} R_{2}(x, y) \leq \bigvee_{y \in U-\{x\}} R_{1}(x, y)$.

(3) This is obvious.

(4) This holds by (3) and Theorem 20(1).

(5) By (1), $\tau_{R_{1} \cup R_{2}} \subseteq \tau_{R_{1}} \cap \tau_{R_{2}}$.

For each $A \in \tau_{R_{1}} \cap \tau_{R_{2}}$, we have $A=R_{1}(A)$ and $A=R_{2}(A)$. By Proposition 18(1),

$$
A=\underline{R_{1}}(A) \cap \underline{R_{2}}(A)=\left(\underline{R_{1} \cup R_{2}}\right)(A) .
$$

Then $A \in \tau_{R_{1} \cup R_{2}}$. Thus $\tau_{R_{1} \cup R_{2}} \supseteq \tau_{R_{1}} \cap \tau_{R_{2}}$.

Hence $\tau_{R_{1} \cup R_{2}}=\tau_{R_{1}} \cap \tau_{R_{2}}$.

Definition 25. Let $R$ be a fuzzy relation on $U$. $R$ is called pseudoconstant if there exists $a \in I$ such that for any $x, y \in U$,

$$
R(x, y)= \begin{cases}1, & x=y \\ a, & x \neq y .\end{cases}
$$

We write $R$ by $a^{*}$ or $a_{U \times U}^{*}$.

That is,

$$
a^{*}(x, y)= \begin{cases}1, & x=y \\ a, & x \neq y\end{cases}
$$

Obviously, every pseudoconstant fuzzy relation is an equivalence fuzzy relation.

Remark 26. (1) For any $a, b \in I, a \leq b$ implies $a^{*} \subseteq b^{*}$.

(2) For each $a \in I, \theta_{a^{*}}=\tau_{a^{*}}$.

(3) $\tau_{0^{*}}=F(U), \tau_{1^{*}}=\{\bar{a}: a \in I\}$.

Remark 27. Let $R$ be a reflexive fuzzy relation on $U$. Then

$$
s_{R}^{*} \subseteq R \subseteq t_{R}^{*}
$$

The following theorem gives the topological structure of reflexive fuzzy approximation spaces.

Theorem 28. Let $(U, R)$ be a reflexive fuzzy approximation space.

(1) Consider $\tau_{R}=\tau_{1^{*}} \cup\left\{A \in F(U): \forall(x, y) \in R_{A}, A(x) \vee\right.$ $A(y) \leq 1-R(x, y)\}$.

(2) Consider $\tau_{t_{R}^{*}} \subseteq \tau_{R} \subseteq \tau_{s_{R}^{*}}$.

(3) For each $a \in I, \tau_{a^{*}}=\tau_{1^{*}} \cup\left\{A \in F(U): R_{A} \neq \emptyset\right.$, $\left.\bigvee_{x \in U} A(x) \leq 1-a\right\}$. 
Proof. (1) This holds by Proposition 17(2), Theorem 20(1), and Remark 26(3).

(2) This holds by Proposition 24(1) and Remark 27.

(3) This holds by (1).

\subsection{Fuzzy Topologies Induced by Preorder Fuzzy}

Approximation Spaces

Theorem 29 (see $[28])$. Let $(U, R)$ be a preorder fuzzy approximation space. Then consider the following.

(1) $\theta_{R}$ is a fuzzy topology on $U$.

(2) $\underline{R}$ is the interior operator of $\theta_{R}$.

(3) $\bar{R}$ is the closure operator of $\theta_{R}$.

Example 30. Let $U=\{x, y, z\}$ and let $R$ be a fuzzy relation on $U$ where

$$
R=\left(\begin{array}{ccc}
1 & 0 & 0 \\
0.7 & 1 & 1 \\
0 & 0.8 & 1
\end{array}\right)
$$

Then $R$ is reflexive. Note that

$$
R(z, y) \wedge R(y, x)=0.7 \not 0=R(z, x) .
$$

Then $R$ is not transitive. So $R$ is not preorder.

Pick

$$
\begin{aligned}
& A=\frac{0}{x}+\frac{0.4}{y}+\frac{1}{z}, \\
& B=\frac{1}{x}+\frac{0.6}{y}+\frac{0}{z} .
\end{aligned}
$$

(1) Since

$$
\begin{gathered}
\underline{R}(A)=\frac{0}{x}+\frac{0.3}{y}+\frac{0.4}{z}, \\
\underline{R}(\underline{R}(A))=\frac{0}{x}+\frac{0.3}{y}+\frac{0.3}{z},
\end{gathered}
$$

we have $\underline{R}(\underline{R}(A)) \neq \underline{R}(A)$. Then $\underline{R}(A) \notin \tau_{R}$. Thus

$$
\theta_{R} \neq \tau_{R}
$$

(2) Obviously, $B^{c}=A$. Note that $R(A) \notin \tau_{R}$ and int $_{\tau_{R}}(B) \in \tau_{R}$. Then

$$
\begin{aligned}
& \operatorname{int}_{\tau_{R}}(A) \neq \underline{R}(A), \\
& \operatorname{cl}_{\tau_{R}}(B) \neq \bar{R}(B) .
\end{aligned}
$$

Thus, $\underline{R}$ is not the interior operator of $\tau_{R}$ and $\bar{R}$ is not the closure operator of $\tau_{R}$.
Example 31. Let $U=\{x, y, z, w\}$ and let $R$ be the fuzzy relation on $U$ where

$$
R=\left(\begin{array}{llll}
0 & 1 & 0 & 0 \\
0 & 0 & 1 & 0 \\
0 & 0 & 0 & 1 \\
1 & 0 & 0 & 0
\end{array}\right)
$$

Then $R$ is neither reflexive nor transitive.

For each $A \in F(U)$, since

$$
\begin{aligned}
& \underline{R}(A)=\frac{A(y)}{x}+\frac{A(z)}{y}+\frac{A(w)}{z}+\frac{A(x)}{w}=B=\bar{R}(A), \\
& \underline{R}(B)=\frac{A(z)}{x}+\frac{A(w)}{y}+\frac{A(x)}{z}+\frac{A(y)}{w}=C=\bar{R}(B), \\
& \underline{R}(C)=\frac{A(w)}{x}+\frac{A(x)}{y}+\frac{A(y)}{z}+\frac{A(z)}{w}=D=\bar{R}(C), \\
& \underline{R}(D)=\frac{A(x)}{x}+\frac{A(y)}{y}+\frac{A(z)}{z}+\frac{A(w)}{w}=A=\bar{R}(D),
\end{aligned}
$$

we have $\underline{R}(\underline{R}(\underline{R}(\underline{R}(A))))=A$. Then $\theta_{R}=F(U)$.

Obviously, $\theta_{R}$ is a fuzzy topology on $U$.

Put $W=x_{0.3} \in P(U)$. Then

$$
\begin{gathered}
\operatorname{cl}_{\theta_{R}}(W)=x_{0.3} \neq w_{0.3}=\bar{R}(W), \\
\operatorname{int}_{\theta_{R}}(W)=x_{0.3} \neq w_{0.3}=\underline{R_{2}}(W) .
\end{gathered}
$$

Thus, $\underline{R}$ is not the interior operator of $\theta_{R}$ and $\bar{R}$ is not the closure operator of $\theta_{R}$.

The following theorem reveals fuzzy relations can be researched by means of topology.

Theorem 32. Let $R_{1}$ and $R_{2}$ be two preorder fuzzy relations on $U$. Then

(1) $\theta_{R_{2}}=\theta_{R_{1}} \Leftrightarrow R_{1}=R_{2}$.

(2) $\theta_{R_{1}} \cap \theta_{R_{2}} \subseteq \theta_{R_{1} \cup R_{2}}$.

Proof

(1) Necessity. Suppose that $\theta_{R_{2}}=\theta_{R_{1}}$. By Remark 7 and Theorem 29(3),

$$
\begin{aligned}
R_{1}(x, y) & =\overline{R_{1}}\left(y_{1}\right)(x)=\operatorname{cl}_{R_{\tau_{1}}}\left(y_{1}\right)(x) \\
& =\operatorname{cl}_{R_{\tau_{2}}}\left(y_{1}\right)(x)=R_{2}(x, y)
\end{aligned}
$$

for any $x, y \in U$. Then $R_{1}=R_{2}$.

Sufficiency. This is obvious.

(2) By Propositions 19 and 24(5),

$$
\theta_{R_{1}} \cap \theta_{R_{2}}=\tau_{R_{1}} \cap \tau_{R_{2}}=\tau_{R_{1} \cup R_{2}} \subseteq \theta_{R_{1} \cup R_{2}} .
$$


Example 33. Let $U=\{x, y, z, w\}$ and let $R_{1}$ and $R_{2}$ be two fuzzy relations on $U$, where

$$
\begin{aligned}
& R_{1}=\left(\begin{array}{llll}
1 & 0 & 1 & 0 \\
0 & 1 & 0 & 1 \\
0 & 1 & 1 & 0 \\
1 & 0 & 0 & 1
\end{array}\right), \\
& R_{2}=\left(\begin{array}{llll}
1 & 0 & 0 & 1 \\
1 & 1 & 0 & 0 \\
0 & 1 & 1 & 0 \\
0 & 0 & 1 & 1
\end{array}\right) .
\end{aligned}
$$

Then $R_{1}$ and $R_{2}$ are reflexive. Note that

$$
\begin{aligned}
& R_{1}(x, z) \wedge R_{1}(z, y)=1 \nless 0=R_{1}(x, y), \\
& R_{2}(x, w) \wedge R_{2}(w, z)=1 \nless 0=R_{2}(x, z) .
\end{aligned}
$$

Then $R_{1}$ and $R_{2}$ are not transitive. So $R_{1}$ and $R_{2}$ are not preorder.

For any $A, B \in F(U)$, since

$$
\begin{aligned}
\underline{R_{1}}(A)= & \frac{A(x) \wedge A(z)}{x}+\frac{A(y) \wedge A(w)}{y} \\
& +\frac{A(y) \wedge A(z)}{z}+\frac{A(x) \wedge A(w)}{w}=A, \\
\underline{R_{2}}(B)= & \frac{B(x) \wedge B(w)}{x}+\frac{B(x) \wedge B(y)}{y} \\
& +\frac{B(y) \wedge B(\mathrm{z})}{z}+\frac{B(z) \wedge B(w)}{w}=B,
\end{aligned}
$$

we have

$$
\begin{aligned}
& A(z) \geq A(x) \geq A(w) \geq A(y) \geq A(z), \\
& B(w) \geq B(x) \geq B(y) \geq B(z) \geq B(w) .
\end{aligned}
$$

So $A(x)=A(y)=A(z)=A(w)$ and $B(x)=B(y)=B(z)=$ $B(w)$.

Thus $\tau_{R_{1}}=\{\bar{a}: a \in I\}=\tau_{R_{2}}$. But $R_{1} \neq R_{2}$.

\section{Fuzzy Topologies versus Fuzzy Approximation Spaces}

As can be seen from Section 4, a reflexive fuzzy approximation space yields a fuzzy topology. In this section, we consider the reverse problem; that is, under which conditions can a fuzzy topology be associated with a fuzzy approximation space which produces the given fuzzy topology?

In what follows, we investigate fuzzy approximation spaces or fuzzy relations induced by fuzzy topologies, introduce the concept of fuzzy approximating spaces, and consider fuzzy topologies which satisfy (CC) axiom.

\subsection{Fuzzy Approximation Spaces Induced by Fuzzy Topologies}

Definition 34. Let $\tau$ be a fuzzy topology on $U$. Define a fuzzy relation $R_{\tau}$ on $U$ by

$$
R_{\tau}(x, y)=\mathrm{cl}_{\tau}\left(y_{1}\right)(x)((x, y) \in U \times U) .
$$

Then $R_{\tau}$ is called the fuzzy relation induced by $\tau$ on $U$ and $\left(U, R_{\tau}\right)$ is called the fuzzy approximation space induced by $\tau$ on $U$.

Theorem 35. Let $\tau$ be a fuzzy topology on $U$ and let $R_{\tau}$ be the fuzzy relation induced by $\tau$ on $U$. Then consider the following.

(1) $R_{\tau}$ is reflexive.

(2)

$$
\underline{R_{\tau}}(A) \subseteq \operatorname{int}_{\tau}(A) \subseteq A \subseteq \mathrm{cl}_{\tau}(A) \subseteq \overline{R_{\tau}}(A) \quad(A \in F(U)) .
$$

Proof. (1) For each $x \in U$,

$$
R_{\tau}(x, x)=\mathrm{cl}_{\tau}\left(x_{1}\right)(x) \geq\left(x_{1}\right)(x)=1 .
$$

Then $R_{\tau}$ is reflexive.

(2) For each $A \in F(U)$, by Remark 1 and Proposition 8(2),

$$
\begin{aligned}
\mathrm{cl}_{\tau}(A) & =\operatorname{cl}_{\tau}\left(\bigcup_{y \in U}\left(A(y) y_{1}\right)\right) \\
& =\bigcup_{y \in U} \mathrm{cl}_{\tau}\left(A(y) y_{1}\right) \\
& =\bigcup_{y \in U} \mathrm{cl}_{\tau}\left(\overline{A(y)} \cap y_{1}\right) \\
& \subseteq \bigcup_{y \in U}\left(\mathrm{cl}_{\tau}(\overline{A(y)}) \cap \mathrm{cl}_{\tau}\left(y_{1}\right)\right) \\
& =\bigcup_{y \in U}\left(\overline{A(y)} \cap \mathrm{cl}_{\tau}\left(y_{1}\right)\right) .
\end{aligned}
$$

Then for each $x \in U$,

$$
\begin{aligned}
\mathrm{cl}_{\tau}(A)(x) & \leq \bigvee_{y \in U}\left(\overline{A(y)}(x) \wedge \mathrm{cl}_{\tau}\left(y_{1}\right)(x)\right) \\
& =\bigvee_{y \in U}\left(A(y) \wedge R_{\tau}(x, y)\right) \\
& =\overline{R_{\tau}}(A)(x) .
\end{aligned}
$$

Thus $\mathrm{cl}_{\tau}(A) \subseteq \overline{R_{\tau}}(A)$.

By Proposition 8(3),

$$
\operatorname{int}_{\tau}(A)=\left(\operatorname{cl}_{\tau}\left(A^{c}\right)\right)^{c} \supseteq\left(\overline{R_{\tau}}\left(A^{c}\right)\right)^{c}=\underline{R_{\tau}}(A) .
$$

Hence

$$
\underline{R_{\tau}}(A) \subseteq \operatorname{int}_{\tau}(A) \subseteq A \subseteq \mathrm{cl}_{\tau}(A) \subseteq \overline{R_{\tau}}(A) .
$$

Theorem 36. Let $R$ be a reflexive fuzzy relation, let $\tau_{R}$ be the fuzzy topology by $R$ on $U$, and let $R_{\tau_{R}}$ be the fuzzy relation induced by $\tau_{R}$ on $U$. If $R$ is transitive, then $R_{\tau_{R}}=R$. 
Proof. For each $(x, y) \in U \times U$, by Remark 7, Proposition 19, and Theorem 29(3),

$$
R(x, y)=\bar{R}\left(y_{1}\right)(x)=\mathrm{cl}_{\theta_{R}}\left(y_{1}\right)(x)=\mathrm{cl}_{\tau_{R}}\left(y_{1}\right)(x) .
$$

Note that $R_{\tau_{R}}(x, y)=\mathrm{cl}_{\tau_{R}}\left(y_{1}\right)(x)$. Then $R_{\tau_{R}}(x, y)=R(x, y)$. Thus

$$
R_{\tau_{R}}=R
$$

5.2. (CC) Axiom. The following condition for a fuzzy topology $\tau$ on $U$ is called (CC) axiom:

(CC) axiom: for any $\lambda \in I$ and $A \in F(U)$,

$$
\mathrm{cl}_{\tau}(\lambda A)=\lambda \mathrm{cl}_{\tau}(A) .
$$

Theorem 37. Let $\tau$ be a fuzzy topology on $U$. If $\tau$ satisfies (CC) axiom, then

(1) $\overline{R_{\tau}}$ is the closure operator of $\tau$.

(2) $\tau$ is Alexandrov.

Proof. (1) For each $A \in F(U)$, by Remark 1 and (CC) axiom,

$$
\begin{aligned}
\mathrm{cl}_{\tau}(A) & =\mathrm{cl}_{\tau}\left(\bigcup_{y \in U}\left(A(y) y_{1}\right)\right) \\
& =\bigcup_{y \in U} \mathrm{cl}_{\tau}\left(A(y) y_{1}\right) \\
& =\bigcup_{y \in U}\left(A(y) \mathrm{cl}_{\tau}\left(y_{1}\right)\right) .
\end{aligned}
$$

Then for each $x \in U$,

$$
\begin{aligned}
\operatorname{cl}_{\tau}(A)(x) & =\bigvee_{y \in U}\left(A(y)(x) \wedge \mathrm{cl}_{\tau}\left(y_{1}\right)(x)\right) \\
& =\bigvee_{y \in U}\left(A(y) \wedge R_{\tau}(x, y)\right) \\
& =\overline{R_{\tau}}(A)(x) .
\end{aligned}
$$

Thus $\overline{R_{\tau}}(A)=\mathrm{cl}_{\tau}(A)$. So $\overline{R_{\tau}}$ is the closure operator of $\tau$.

(2) For each $A \in F(U)$, by (CC) axiom and Theorem 37(1), $\bar{R}\left(A^{c}\right)=c l_{\tau}\left(A^{c}\right)$. Then $\underline{R}(A)=\operatorname{int}_{\tau}(A)$.

Let $\left\{A_{j}: j \in J\right\} \subseteq \tau$. Then $\operatorname{int}\left(A_{j}\right)=A_{j}$ for each $j \in J$. Note that $\underline{R}\left(A_{j}\right)=\operatorname{int}_{\tau}\left(A_{j}\right)$. Then $A_{j}=\underline{R}\left(A_{j}\right)$. By Proposition 8(5),

$$
\bigcap_{j \in J} A_{j}=\bigcap_{j \in J} \underline{R}\left(A_{j}\right)=\underline{R}\left(\bigcap_{j \in J} A_{j}\right)=\operatorname{int}_{\tau}\left(\bigcap_{j \in J} A_{j}\right) .
$$

So $\bigcap_{j \in J} A_{j} \in \tau$. Hence $\tau$ is Alexandrov.

For any $\mathscr{A}, \mathscr{B} \subseteq F(U)$, define

$$
\begin{aligned}
& \mathscr{A} \wedge \mathscr{B}=\{A \cap B: A \in \mathscr{A}, B \in \mathscr{B}\}, \\
& \mathscr{A} \vee \mathscr{B}=\{A \cup B: A \in \mathscr{A}, B \in \mathscr{B}\},
\end{aligned}
$$

$$
\begin{aligned}
& \cap \mathscr{A}=\bigcap_{A \in \mathscr{A}} A, \\
& \cup \mathscr{A}=\bigcup_{A \in \mathscr{A}} A .
\end{aligned}
$$

Example 38. Let $U=\{x, y, z\}$. Put $\tau=\bigcup_{i=1}^{5} \tau_{i}$, where

$$
\begin{gathered}
\tau_{1}=\{\bar{a}: a \in I\}, \\
\tau_{2}=\left\{A_{a b}: a \in[0.5,0.7], b \in I\right\} \\
\text { with } A_{a b}=\frac{a}{x}+\frac{0.7}{y}+\frac{b}{z},
\end{gathered}
$$

$$
\tau_{3}=\left\{B_{a b}: a \in[0.5,0.7], b \in I\right\}
$$$$
\text { with } B_{a b}=\frac{0.5}{x}+\frac{a}{y}+\frac{b}{z} \text {, }
$$

$$
\tau_{4}=\left\{C_{a b}: a, b \in I\right\}
$$

with $C_{a b}=\frac{a}{x}+\frac{a}{y}+\frac{b}{z}$,

$$
\begin{array}{r}
\tau_{5}=\left\{D_{a b c}: 0.5 \leq a \leq b \leq 0.7, c \in I\right\} \\
\text { with } D_{a b c}=\frac{a}{x}+\frac{b}{y}+\frac{c}{z} .
\end{array}
$$

(1) For any $\mathscr{C}_{i} \subseteq \tau_{i}$,

$$
\bigcap \mathscr{C}_{i} \subseteq \tau_{i}, \quad \bigcup \mathscr{C}_{i} \subseteq \tau_{i} \quad(i=1,2,3,4,5)
$$

For any $d \in I, A_{a b} \in \tau_{2}$ and $B_{a b} \in \tau_{3}$,

$$
\bar{d} \cap A_{a b}= \begin{cases}\frac{d}{x}+\frac{d}{y}+\frac{d \wedge b}{z} \in \tau_{4}, & d \in[0,0.5), \\ \frac{a}{x}+\frac{d}{y}+\frac{d \wedge b}{z} \in \tau_{5}, & a<d, d \in[0.5,0.7), \\ \frac{d}{x}+\frac{d}{y}+\frac{d \wedge b}{z} \in \tau_{4}, & a \geq d, d \in[0.5,0.7), \\ \frac{a}{x}+\frac{0.7}{y}+\frac{d \wedge b}{z} \in \tau_{2}, & d \in[0.7,1],\end{cases}
$$$$
\bar{d} \cup A_{a b}= \begin{cases}\frac{d \vee a}{x}+\frac{0.7}{y}+\frac{d \vee b}{z} \in \tau_{2}, & d \in[0,0.7), \\ \frac{d}{x}+\frac{d}{y}+\frac{d \vee b}{z} \in \tau_{4}, & d \in[0.7,1]\end{cases}
$$$$
\bar{d} \cap B_{a b}= \begin{cases}\frac{d}{x}+\frac{d}{y}+\frac{d \wedge b}{z} \in \tau_{4}, & d \in[0,0.5), \\ \frac{0.5}{x}+\frac{d \wedge a}{y}+\frac{d \wedge b}{z} \in \tau_{3}, & d \in[0.5,1]\end{cases}
$$ 


$$
\bar{d} \cup B_{a b}= \begin{cases}\frac{0.5}{x}+\frac{a}{y}+\frac{d \vee b}{z} \in \tau_{3}, & d \in[0,0.5), \\ \frac{d}{x}+\frac{a}{y}+\frac{d \vee b}{z} \in \tau_{5}, & a>d, d \in[0.5,0.7), \\ \frac{d}{x}+\frac{d}{y}+\frac{d \vee b}{z} \in \tau_{4}, & a \leq d, d \in[0.5,0.7), \\ \frac{d}{x}+\frac{d}{y}+\frac{d \vee b}{z} \in \tau_{4}, & d \in[0.7,1] .\end{cases}
$$

Then

$$
\begin{gathered}
\tau_{1} \wedge \tau_{2} \subseteq \tau_{4} \cup \tau_{5} \cup \tau_{2} \subseteq \tau, \\
\tau_{1} \vee \tau_{2} \subseteq \tau_{2} \cup \tau_{4} \subseteq \tau, \\
\tau_{1} \wedge \tau_{3} \subseteq \tau_{4} \cup \tau_{3} \subseteq \tau, \\
\tau_{1} \vee \tau_{3} \subseteq \tau_{3} \cup \tau_{5} \cup \tau_{4} \subseteq \tau .
\end{gathered}
$$

Similarly, we can prove that

$$
\begin{gathered}
\tau_{1} \wedge \tau_{4} \subseteq \tau_{4} \subseteq \tau, \\
\tau_{1} \vee \tau_{4} \subseteq \tau_{4} \subseteq \tau ; \\
\tau_{1} \wedge \tau_{5} \subseteq \tau_{4} \cup \tau_{5} \subseteq \tau, \\
\tau_{1} \vee \tau_{5} \subseteq \tau_{5} \cup \tau_{4} \subseteq \tau ; \\
\tau_{2} \wedge \tau_{3} \subseteq \tau_{3} \subseteq \tau, \\
\tau_{2} \vee \tau_{3} \subseteq \tau_{2} \subseteq \tau ; \\
\tau_{2} \wedge \tau_{4} \subseteq \tau_{4} \cup \tau_{5} \cup \tau_{2} \subseteq \tau, \\
\tau_{2} \vee \tau_{4} \subseteq \tau_{2} \cup \tau_{4} \subseteq \tau ; \\
\tau_{2} \wedge \tau_{5} \subseteq \tau_{5} \subseteq \tau, \\
\tau_{2} \vee \tau_{5} \subseteq \tau_{2} \subseteq \tau ; \\
\tau_{3} \wedge \tau_{4} \subseteq \tau_{4} \cup \tau_{3} \subseteq \tau, \\
\tau_{3} \vee \tau_{4} \subseteq \tau_{3} \cup \tau_{5} \cup \tau_{4} \subseteq \tau ; \\
\tau_{3} \wedge \tau_{5} \subseteq \tau_{3} \subseteq \tau, \\
\tau_{3} \vee \tau_{5} \subseteq \tau_{5} \subseteq \tau ; \\
\tau_{4} \wedge \tau_{5} \subseteq \tau_{4} \cup \tau_{5} \subseteq \tau, \\
\tau_{4} \vee \tau_{5} \subseteq \tau_{5} \cup \tau_{4} \subseteq \tau
\end{gathered}
$$

(2) (i) For each $a \in I, \bar{a} \in \tau_{1} \subseteq \tau$.

(ii) Let $\mathscr{A} \subseteq \tau$ and $\mathscr{A} \neq \emptyset$. Then there exist $\mathscr{A}_{1}, \mathscr{A}_{2}, \mathscr{A}_{3}$, $\mathscr{A}_{4}$, and $\mathscr{A}_{5}$ such that $\mathscr{A}=\bigcup_{i=1}^{5} \mathscr{A}_{i}$ and

$$
\emptyset \neq \mathscr{A}_{i} \subseteq \tau_{i} \quad \text { or } \quad \mathscr{A}_{i}=\emptyset \quad(i=1,2,3,4,5) .
$$

If $\mathscr{A}_{i} \subseteq \tau_{i}$, then by $\left(1^{*}\right)$,

$$
\bigcap_{A \in \mathscr{A}_{i}} A=\bigcap \mathscr{A}_{i} \in \tau_{\mathrm{i}}, \quad \bigcup_{A \in \mathscr{A}_{i}} A=\bigcup \mathscr{A}_{i} \in \tau_{i} .
$$

By $\left(2^{*}\right)$ and $\left(3^{*}\right)$,

$$
\bigcap_{A \in \mathscr{A}} A=\bigcap_{i=1}^{5}\left(\bigcap_{A \in \mathscr{A}_{i}} A\right) \in \tau, \quad \bigcup_{A \in \mathscr{A}} A=\bigcup_{i=1}^{5}\left(\bigcup_{A \in \mathscr{A}_{i}} A\right) \in \tau \text {. }
$$

Thus, $\tau$ is an Alexandrov fuzzy topology on $U$.

(3) Put $\lambda=0.4$ and $A=x_{1} \in P(U)$. Then $\lambda A=x_{0.4}$. Note that

(i) $\bigcap\left\{B \in \tau_{1}^{c}: B \supseteq x_{0.4}\right\}=\overline{0.4}, \bigcap\left\{B \in \tau_{1}^{c}: B \supseteq A\right\}=\overline{1}$;

(ii) $\bigcap\left\{B \in \tau_{2}^{c}: B \supseteq x_{0.4}\right\}=(0.4 / x)+(0.3 / y)+(0 / z)$, $\left\{B \in \tau_{2}^{c}: B \supseteq A\right\}=\emptyset$

(iii) $\bigcap\left\{B \in \tau_{3}^{c}: B \supseteq x_{0.4}\right\}=(0.5 / x)+(0.3 / y)+(0 / z)$, $\left\{B \in \tau_{3}^{c}: B \supseteq A\right\}=\emptyset$

(iv) $\bigcap\left\{B \in \tau_{4}^{c}: B \supseteq x_{0.4}\right\}=(0.4 / x)+(0.4 / y)+(0 / z)$, $\bigcap\left\{B \in \tau_{4}^{c}: B \supseteq A\right\}=(1 / x)+(1 / y)+(0 / z)$

(v) $\bigcap\left\{B \in \tau_{5}^{c}: B \supseteq x_{0.4}\right\}=(0.4 / x)+(0.3 / y)+(0 / z)$, $\left\{B \in \tau_{5}^{c}: B \supseteq A\right\}=\emptyset$.

Then

$$
\begin{gathered}
\mathrm{cl}_{\tau}(\lambda A)=\mathrm{cl}_{\tau}\left(x_{0.4}\right)=\frac{0.4}{x}+\frac{0.3}{y}+\frac{0}{z}, \\
\operatorname{cl}_{\tau}(A)=\frac{1}{x}+\frac{1}{y}+\frac{0}{z} .
\end{gathered}
$$

Then

$$
\begin{aligned}
\mathrm{cl}_{\tau}(\lambda A) & =\frac{0.4}{x}+\frac{0.3}{y}+\frac{0}{z} \neq \frac{0.4}{x}+\frac{0.4}{y}+\frac{0}{z} \\
& =\lambda \mathrm{cl}_{\tau}(A) .
\end{aligned}
$$

Thus, $\tau$ does not satisfy (CC) axiom.

Theorem 39. Let $R$ be a preorder fuzzy relation on $U$. Then $\tau_{R}$ satisfies (CC) axiom.

Proof. For any $\lambda \in I$ and $A \in F(U)$, by Theorem 29(3) and Proposition 8(6),

$$
\mathrm{cl}_{\tau_{R}}(\lambda A)=\bar{R}(\lambda A)=\lambda \bar{R}(A)=\lambda \mathrm{cl}_{\tau_{R}}(A) .
$$

Thus $\tau_{R}$ satisfies (CC) axiom.

Theorem 40. Let $\tau$ be a fuzzy topology on $U$, let $R_{\tau}$ be the fuzzy relation induced by $\tau$ on $U$, and let $\tau_{R_{\tau}}$ be the fuzzy topology induced by $R_{\tau}$ on $U$. Then

$$
\tau_{R_{\tau}}=\tau \text { iff } \tau \text { satisfies (CC) axiom. }
$$

Proof

Necessity. For each $A \in F(U)$, by Theorem 35(2), $R_{\tau}(A) \subseteq$ $\operatorname{int}_{\tau}(A)$. By Theorems 35(1) and 20(2),

$$
\operatorname{int}_{\tau}(A)=\operatorname{int}_{\tau_{R_{\tau}}}(A) \subseteq \underline{R_{\tau}}(A) .
$$

Then

$$
\operatorname{int}_{\tau}(A)=\underline{R_{\tau}}(A) \quad(A \in U) .
$$

Thus $\underline{R}$ is the closure operator of $\tau$. 
Sufficiency. By Theorem 35(1), $R_{\tau}$ is reflexive. For any $x, y, z \in$ $U$, put $\mathrm{cl}_{\tau}\left(z_{1}\right)(y)=\lambda$. By Remark 1 and Proposition 8(2),

$$
\begin{aligned}
\lambda \mathrm{cl}_{\tau}\left(y_{1}\right) & =\mathrm{cl}_{\tau}\left(\lambda y_{1}\right) \\
& =\operatorname{cl}_{\tau}\left(\mathrm{cl}_{\tau}\left(z_{1}\right)(y) y_{1}\right) \\
& \subseteq \mathrm{cl}_{\tau}\left(\bigcup_{t \in U}\left(\mathrm{cl}_{\tau}\left(z_{1}\right)(t) t_{1}\right)\right) \\
& =\operatorname{cl}_{\tau}\left(\operatorname{cl}_{\tau}\left(z_{1}\right)\right)=\mathrm{cl}_{\tau}\left(z_{1}\right) .
\end{aligned}
$$

Then

$$
\begin{aligned}
R_{\tau}(x, y) \wedge R_{\tau}(y, z) & =\operatorname{cl}_{\tau}\left(y_{1}\right)(x) \wedge \mathrm{cl}_{\tau}\left(z_{1}\right)(y) \\
& =\operatorname{cl}_{\tau}\left(y_{1}\right)(x) \wedge \lambda \\
& =\lambda \wedge \mathrm{cl}_{\tau}\left(y_{1}\right)(x) \\
& =\left(\lambda \mathrm{cl}_{\tau}\left(y_{1}\right)\right)(x) \\
& \leq \mathrm{cl}_{\tau}\left(z_{1}\right)(x)=R_{\tau}(x, z)
\end{aligned}
$$

Then $R_{\tau}$ is transitive.

So $R_{\tau}$ is preorder. For each $A \in F(U)$, by Proposition 19 and Theorem 29(3)

$$
\mathrm{cl}_{\tau_{R_{\tau}}}(A)=\mathrm{cl}_{\theta_{R_{\tau}}}(A)=\overline{R_{\tau}}(A) .
$$

By (CC) axiom and Theorem 37(1), $\overline{R_{\tau}}(A)=\mathrm{cl}_{\tau}(A)$. So $\mathrm{cl}_{\tau_{R_{\tau}}}(A)=\mathrm{cl}_{\tau}(A)$. Thus

$$
\tau_{R_{\tau}}=\tau
$$

Theorem 41. Let $\tau$ be a fuzzy topology on $U$. Then the following are equivalent.

(1) $\tau$ satisfies (CC) axiom.

(2) For any $\lambda \in I$ and $A \in F(U)$,

$$
\operatorname{int}_{\tau}(\bar{\lambda} \cup A)=\bar{\lambda} \cup \operatorname{int}_{\tau}(A) .
$$

(3) There exists a preorder fuzzy relation $\rho$ on $U$ such that $\bar{\rho}$ is the closure operator of $\tau$.

(4) There exists a preorder fuzzy relation $\rho$ on $U$ such that $\rho$ is the interior operator of $\tau$.

(5) $\overline{R_{\tau}}$ is the closure operator of $\tau$.

(6) $R_{\tau}$ is the interior operator of $\tau$.

Proof. (1) $\Leftrightarrow$ (2). This is obvious.

$(1) \Rightarrow(3)$. Suppose that $\tau$ satisfies (CC) axiom. Pick $\rho=$ $R_{\tau}$. By Theorem 37(1), $\bar{\rho}$ is the closure operator of $\tau$. By Theorem 16(1), $\bar{\rho}$ is preorder.

$(3) \Rightarrow(4)$. Let $\bar{\rho}$ be the closure operator of $\tau$ for some preorder fuzzy relation $\rho$ on $U$. For each $A \in F(U)$, by Proposition 8(3),

$$
\underline{\rho}(A)=\left(\bar{\rho}\left(A^{c}\right)\right)^{c}=\left(\mathrm{cl}_{\tau}\left(A^{c}\right)\right)^{c}=\operatorname{int}_{\tau}(A) .
$$

Thus, $\rho$ is the interior operator of $\tau$.
$(4) \Rightarrow(6)$. Let $\rho$ be the interior operator of $\tau$ for some preorder fuzzy relation $\rho$ on $U$.

For $(x, y) \in U \times U$, by Remark 7,

$$
\begin{aligned}
\rho(x, y) & =1-\underline{\rho}\left(\left(y_{1}\right)^{c}\right)(x) \\
& =1-\operatorname{int}_{\tau}\left(\left(y_{1}\right)^{c}\right)(x) \\
& =\operatorname{cl}_{\tau}\left(y_{1}\right)(x)=R_{\tau}(x, y) .
\end{aligned}
$$

Then $\rho=R_{\tau}$. Note that $\rho$ is the interior operator of $\tau$. Then $R_{\tau}$ is the interior operator of $\tau$.

$(6) \Rightarrow(5)$ holds by Proposition $8(3)$.
$(5) \Rightarrow$ (1). For any $a \in I$ and $A \in F(U)$, by Proposition 8(6),

$$
\mathrm{cl}_{\tau}(\lambda A)=\overline{R_{\tau}}(\lambda A)=\lambda \overline{R_{\tau}}(A)=\lambda \mathrm{cl}_{\tau}(A) .
$$

Thus $\tau$ satisfies (CC) axiom.

The following theorem reveals that we can research fuzzy relations or fuzzy approximation spaces by means topology and vice versa.

Theorem 42. Let

$$
\begin{gathered}
\Sigma=\{R: R \text { is a preorder fuzzy relation on } U\}, \\
\Gamma=\{\tau: \tau \text { is a fuzzy topology on } U \\
\text { satisfying }(C C) \text { axiom }\} .
\end{gathered}
$$

Then there exists a one-to-one correspondence between $\Sigma$ and $\Gamma$.

Proof. Two mappings $f: \Sigma \rightarrow \Gamma$ and $g: \Gamma \rightarrow \Sigma$ are defined as follows:

$$
\begin{array}{ll}
f(R)=\tau_{R} & (R \in \Sigma), \\
g(\tau)=R_{\tau} & (\tau \in \Gamma) .
\end{array}
$$

By Theorem 36,

$$
g \circ f=i_{\Sigma}
$$

where $g \circ f$ is the composition of $f$ and $g$ and $i_{\Sigma}$ is the identity mapping on $\Gamma$.

By Theorem 40,

$$
f \circ g=i_{\Gamma}
$$

where $f \circ g$ is the composition of $g$ and $f$ and $i_{\Gamma}$ is the identity mapping on $\Sigma$.

Hence $f$ and $g$ are two one-to-one correspondences. This prove that there exists a one-to-one correspondence between $\Sigma$ and $\Gamma$.

5.3. Fuzzy Approximating Spaces. Below, we introduce the concept of fuzzy approximating spaces, which enable us to study fuzzy relations or fuzzy approximation spaces by means of topology. 
Definition 43. Let $(U, \sigma)$ be a fuzzy topological space. If there exists a reflexive fuzzy relation $R$ on $U$ such that $\tau_{R}=\sigma$, then $(U, \sigma)$ is called a fuzzy approximating space.

Theorem 44. Let $(U, \tau)$ be a fuzzy topological space. If one of the following conditions satisfies, then $(U, \tau)$ is a fuzzy approximating space.

(1) $\tau$ satisfies (CC) axiom.

(2) For any $\lambda \in I$ and $A \in F(U)$,

$$
\operatorname{int}_{\tau}(\bar{\lambda} \cup A)=\bar{\lambda} \cup \operatorname{int}_{\tau}(A) \text {. }
$$

(3) There exists a preorder fuzzy relation $R$ on $U$ such that $\bar{R}$ is the closure operator of $\tau$.

(4) There exists a preorder fuzzy relation $R$ on $U$ such that $\underline{R}$ is the interior operator of $\tau$.

(5) $\overline{R_{\tau}}$ is the closure operator of $\tau$.

(6) $R_{\tau}$ is the interior operator of $\tau$.

Proof. These hold by Theorems 40 and 41 .

By Remark 26, Theorems 39 and 44, we have the following example.

Example 45. $\{\bar{a}: a \in I\}$ is a fuzzy approximating space.

Theorem 46. Let $(U, \tau)$ be a fuzzy topological space. Then $(U, \tau)$ is a fuzzy approximating space if and only if there exist a reflexive fuzzy relation $R$ such that

$\tau=\tau_{1 *}$

$$
\cup\left\{A \in F(U): \forall(x, y) \in R_{A}, A^{c}(x) \wedge A^{c}(y) \geq R(x, y)\right\} .
$$

Proof. This holds by Proposition 17(2), Theorems 20(1) and 28.

\section{Fuzzy Pseudoclosure Operators}

In this section, we will define the concept of fuzzy pseudoclosure operators induced by a fuzzy topology and investigate their properties.

For any $\lambda \in I, X \in 2^{U}$, we define

$$
(\lambda X)(x)= \begin{cases}\lambda, & x \in X \\ 0, & x \in U-X\end{cases}
$$

Denote

$$
E(U)=\left\{\lambda X: \lambda \in I, X \in 2^{U}\right\} .
$$

Obviously, $E(U) \subseteq F(U)$.

Definition 47. Let $\tau$ be a fuzzy topology on $U$. Define

$$
S_{\tau}(A)=\bigcup_{\lambda \in I} \mathrm{cl}_{\tau}\left(\lambda A_{\lambda}\right) \quad(A \in F(U)) .
$$

Then $S_{\tau}: F(U) \rightarrow F(U)$ is called the fuzzy pseudo-closure operator induced by $\tau$.
Theorems 48(5) and 49 below illustrate the meaning on fuzzy pseudoclosure operators.

Theorem 48. Let $\tau$ be a fuzzy topology on $U$ and let $S_{\tau}$ be the fuzzy pseudo-closure operator induced $\tau$. Then for any $A, B \in$ $F(U)$, consider the following.

(1) Consider $S_{\tau}(\overline{0})=\overline{0}$.

(2) Consider $A \subseteq S_{\tau}(A) \subseteq \operatorname{cl}_{\tau}(A)$.

(3) Consider $S_{\tau}(A \cup B) \supseteq S_{\tau}(A) \cup S_{\tau}(B)$. Consider $S_{\tau}(A \cap$ $B) \subseteq S_{\tau}(A) \cap S_{\tau}(B)$.

(4) Consider $A \in \tau^{c} \Rightarrow S_{\tau}(A)=A$.

(5) Consider $S_{\tau}$ coincides with $\mathrm{cl}_{\tau}$ as operators from $E(U)$ to $F(U)$.

Proof. (1) For any $\lambda \in I$ and $x \in U$, since

$$
\left(\lambda \overline{0}_{\lambda}\right)(x)=\lambda \wedge \overline{0}_{\lambda}(x)= \begin{cases}0 \wedge 1=0, & \lambda=0, \\ \lambda \wedge 0=0, & \lambda \in(0,1] .\end{cases}
$$

We have $\lambda \overline{0}_{\lambda}=\overline{0}$. Thus

$$
S_{\tau}(\overline{0})=\bigcup_{\lambda \in I} \mathrm{cl}_{\tau}\left(\lambda \overline{0}_{\lambda}\right)=\bigcup_{\lambda \in I} \mathrm{cl}_{\tau}(\overline{0})=\overline{0}
$$

(2) By $A=\bigcup_{\lambda \in I} \lambda A_{\lambda}$,

$$
A=\bigcup_{\lambda \in I} \lambda A_{\lambda} \subseteq \bigcup_{\lambda \in I} \mathrm{cl}_{\tau}\left(\lambda A_{\lambda}\right)=S_{\tau}(A),
$$

$$
S_{\tau}(A)=\bigcup_{\lambda \in I} \mathrm{cl}_{\tau}\left(\lambda A_{\lambda}\right) \subseteq \mathrm{cl}_{\tau}\left(\bigcup_{\lambda \in I} \lambda A_{\lambda}\right)=\mathrm{cl}_{\tau}(A) .
$$

(3) For any $A, B \in F(U), \lambda \in I$, and $x \in U$, put

$$
\begin{aligned}
& C(x)= \begin{cases}1, & x \in A_{\lambda}, \\
0, & x \in U-A_{\lambda},\end{cases} \\
& D(x)= \begin{cases}1, & x \in B_{\lambda}, \\
0, & x \in U-B_{\lambda} .\end{cases}
\end{aligned}
$$

Obviously, $\lambda A_{\lambda}=\bar{\lambda} \cap C, \lambda B_{\lambda}=\bar{\lambda} \cap D$. Then

$$
\begin{aligned}
& \lambda\left(A_{\lambda} \cup B_{\lambda}\right)=\bar{\lambda} \cap(C \cup D), \\
& \lambda\left(A_{\lambda} \cap B_{\lambda}\right)=\bar{\lambda} \cap(C \cap D) .
\end{aligned}
$$

We can easily prove that

$$
\begin{aligned}
& (A \cup B)_{\lambda} \supseteq A_{\lambda} \cup B_{\lambda}, \\
& (A \cap B)_{\lambda}=A_{\lambda} \cap B_{\lambda} .
\end{aligned}
$$


Thus

$$
\begin{aligned}
S_{\tau}(A & \cup B) \\
& =\bigcup_{\lambda \in I} \mathrm{cl}_{\tau}\left(\lambda(A \cup B)_{\lambda}\right) \\
& \supseteq \bigcup_{\lambda \in I} \mathrm{cl}_{\tau}\left(\lambda\left(A_{\lambda} \cup B_{\lambda}\right)\right) \\
& =\bigcup_{\lambda \in I} \mathrm{cl}_{\tau}(\bar{\lambda} \cap(C \cup D)) \\
& =\bigcup_{\lambda \in I} \mathrm{cl}_{\tau}((\bar{\lambda} \cap C) \cup(\bar{\lambda} \cap D)) \\
& =\bigcup_{\lambda \in I}\left(\mathrm{cl}_{\tau}(\bar{\lambda} \cap C) \cup \mathrm{cl}_{\tau}(\bar{\lambda} \cap D)\right) \\
& =\left(\bigcup_{\lambda \in I} \mathrm{cl}_{\tau}(\bar{\lambda} \cap C)\right) \cup\left(\bigcup_{\lambda \in I} \mathrm{cl}_{\tau}(\bar{\lambda} \cap D)\right) \\
& =\left(\bigcup_{\lambda \in I} \mathrm{cl}_{\tau}\left(\lambda A_{\lambda}\right)\right) \cup\left(\bigcup_{\lambda \in I} \mathrm{cl}_{\tau}\left(\lambda B_{\lambda}\right)\right) \\
= & S_{\tau}(A) \cup S_{\tau}(B),
\end{aligned}
$$

$S_{\tau}(A \cap B)$

$$
\begin{aligned}
& =\bigcap_{\lambda \in I} \mathrm{cl}_{\tau}\left(\lambda(A \cap B)_{\lambda}\right) \\
& =\bigcup_{\lambda \in I} \mathrm{cl}_{\tau}\left(\lambda\left(A_{\lambda} \cap B_{\lambda}\right)\right) \\
& =\bigcup_{\lambda \in I} \mathrm{cl}_{\tau}(\bar{\lambda} \cap(C \cap D)) \\
& =\bigcup_{\lambda \in I} \mathrm{cl}_{\tau}((\bar{\lambda} \cap C) \cap(\bar{\lambda} \cap D)) \\
& \subseteq \bigcup_{\lambda \in I}\left(\mathrm{cl}_{\tau}(\bar{\lambda} \cap C) \cap \mathrm{cl}_{\tau}(\bar{\lambda} \cap D)\right) \\
& \subseteq\left(\bigcup_{\lambda \in I} \mathrm{cl}_{\tau}(\bar{\lambda} \cap C)\right) \cap\left(\bigcup_{\lambda \in I} \mathrm{cl}_{\tau}(\bar{\lambda} \cap D)\right) \\
& =\left(\bigcup_{\lambda \in I} \mathrm{cl}_{\tau}\left(\lambda A_{\lambda}\right)\right) \cap\left(\bigcup_{\lambda \in I} \mathrm{cl}_{\tau}\left(\lambda B_{\lambda}\right)\right) \\
& =S_{\tau}(A) \cap S_{\tau}(B) .
\end{aligned}
$$

(4) By (2),

$$
\mathrm{cl}_{\tau}(A) \subseteq S\left(\mathrm{cl}_{\tau}(A)\right) \subseteq \mathrm{cl}_{\tau}\left(\mathrm{cl}_{\tau}(A)\right)=\mathrm{cl}_{\tau}(A) .
$$

Then

$$
S_{\tau}(A)=S_{\tau}\left(\mathrm{cl}_{\tau}(A)\right)=\mathrm{cl}_{\tau}(A)=A
$$

(5) Let $A \in E(U)$. Then there exist $\lambda \in I$ and $X \in 2^{U}$ such that $A=\lambda X$. (i) If $\lambda \neq 0$, then for each $x \in U$,

$$
\begin{aligned}
A_{\lambda}(x) & =(\lambda X)_{\lambda}(x) \\
& =\left\{\begin{array}{ll}
1, & (\lambda X)(x) \geq \lambda \\
0, & (\lambda X)(x)<\lambda
\end{array}= \begin{cases}1, & x \in X, \\
0, & x \in U-X .\end{cases} \right.
\end{aligned}
$$

Thus $A_{\lambda}=X$. So

$$
\begin{aligned}
S_{\tau}(A) & =\bigcup_{\mu \in I} \mathrm{cl}_{\tau}\left(\mu A_{\mu}\right) \\
& \supseteq \mathrm{cl}_{\tau}\left(\lambda A_{\lambda}\right)=\mathrm{cl}_{\tau}(\lambda X) \\
& =\mathrm{cl}_{\tau}(A) .
\end{aligned}
$$

Note that $S_{\tau}(A) \subseteq \operatorname{cl}(A)$ by (2). Then $S_{\tau}(A)=\operatorname{cl}_{\tau}(A)$. (ii) If $\lambda=0$, then $A=\overline{0}$. By (1), $S_{\tau}(\overline{0})=\overline{0}$.

Thus

$$
S_{\tau}(A)=\mathrm{cl}_{\tau}(A) \quad(A \in E(U)) .
$$

Hence

$S_{\tau}$ coincides with $\mathrm{cl}_{\tau}$ as operators from $E(U)$ to $F(U)$.

Theorem 49. Let $(U, R)$ be a reflexive fuzzy approximation space. Then

$$
S_{\tau_{R}}(A)=\bar{R}(A)(A \in E(U)),
$$

where $\tau_{R}$ is the fuzzy topology induced by $(U, R)$.

Proof. For each $A \in E(U)$, by Proposition 19 and Theorem 29(3),

$$
\mathrm{cl}_{\tau_{R}}(A)=\mathrm{cl}_{\theta_{R}}(A)=\bar{R}(A) .
$$

By Theorem 48(5), $S_{\tau_{R}}(A)=\mathrm{cl}_{\tau_{R}}(A)$. Thus

$$
S_{\tau_{R}}(A)=\bar{R}(A) .
$$

\section{Conclusions}

In this paper, relationships between fuzzy approximation spaces and fuzzy topology were discussed. We proved that there exists a one-to-one correspondence between the set of all preorder fuzzy relations and the set of all fuzzy topologies which satisfy (CC) axiom. We presented the concept of pseudoconstant fuzzy relations and gave topological structures of fuzzy approximation spaces by using them. We introduced the concept of fuzzy approximating spaces and obtained decision conditions that a fuzzy topological space is a fuzzy approximating space.

The results of this paper may have at least the following functions. Fuzzy relations can be researched by means of 
topology or lower and upper sets (see Theorems 15, 16, and 32). The concept of fuzzy approximating spaces and the fact that there exists a one-to-one correspondence between the set of all preorder fuzzy relations and the set of all fuzzy topologies satisfying (CC) axiom enable us to study fuzzy relations or fuzzy approximation spaces by means of topology (see Theorem 42 and Definition 43). Separation axioms can provide means to distinguish various fuzzy rough sets and give more local information or knowledge (see Theorem 23). We hope that one can find applications of topological structures of fuzzy approximation spaces for real life problems.

\section{Conflict of Interests}

The authors declare that there is no conflict of interests regarding the publication of this paper.

\section{Acknowledgments}

The authors would like to thank the editors and the anonymous reviewers for their valuable comments and suggestions which have helped immensely in improving the quality of the paper. This work is supported by the National Natural Science Foundation of China (no. 11371003), the Natural Science Foundation of Guangxi (no. 2011GXNSFA018154, 2012GXNSFGA060003), the Science and Technology Foundation of Guangxi (no. 10169-1), Guangxi Scientific Research Project (no. 201012MS274), Guangxi HCIC lab Open Fund (no. HCIC201306, HCIC201303), and the Science Research Project 2013 of the China-ASEAN Study Center (Guangxi Science Experiment Center) of Guangxi University for Nationalities (no. KT201310).

\section{References}

[1] Z. Pawlak, "Rough sets," International Journal of Computer and Information Sciences, vol. 11, no. 5, pp. 341-356, 1982.

[2] Z. Pawlak, Rough Sets: Theoretical Aspects of Reasoning about Data, Kluwer Academic Publishers, Dordrecht, The Netherlands, 1991.

[3] Z. Pawlak and A. Skowron, "Rudiments of rough sets," Information Sciences, vol. 177, no. 1, pp. 3-27, 2007.

[4] Z. Pawlak and A. Skowron, "Rough sets: some extensions," Information Sciences, vol. 177, no. 1, pp. 28-40, 2007.

[5] Z. Pawlak and A. Skowron, "Rough sets and Boolean reasoning," Information Sciences, vol. 177, no. 1, pp. 41-73, 2007.

[6] A. Skowron and J. Stepaniuk, "Tolerance approximation spaces," Fundamenta Informaticae, vol. 27, no. 2-3, pp. 245-253, 1996.

[7] R. Slowinski and D. Vanderpooten, "Similarity relation as a basis for rough approximations," ICS Research Report, vol. 53, pp. 249-250, 1995.

[8] G. Liu and W. Zhu, "The algebraic structures of generalized rough set theory," Information Sciences, vol. 178, no. 21, pp. 41054113, 2008.

[9] Y. Y. Yao, "Constructive and algebraic methods of the theory of rough sets," Information Sciences, vol. 109, no. 1-4, pp. 21-47, 1998.
[10] W. Zhang, W. Wu, J. Liang, and D. Li, Rough Sets Theorey and Methods, Chinese Scientific Publishers, Beijing, China, 2001.

[11] D. Dubois and H. Prade, "Rough fuzzy sets and fuzzy rough sets," International Journal of General Systems, vol. 17, pp. 191208, 1990.

[12] L. I. Kuncheva, "Fuzzy rough sets: application to feature selection," Fuzzy Sets and Systems, vol. 51, no. 2, pp. 147-153, 1992.

[13] S. Nanda and S. Majumdar, "Fuzzy rough sets," Fuzzy Sets and Systems, vol. 45, no. 2, pp. 157-160, 1992.

[14] A. M. Radzikowska and E. E. Kerre, "A comparative study of fuzzy rough sets," Fuzzy Sets and Systems, vol. 126, no. 2, pp. 137$155,2002$.

[15] H. Thiele, "Fuzzy rough sets versus rough fuzzy sets: an interpretation and a comparative study using concepts of modal logics," in Proceedings of the 5th European Congress on Intelligent Technigues and Soft Computing, pp. 159-167, Aachen, Germany, September 1997.

[16] W.-Z. Wu, J.-S. Mi, and W.-X. Zhang, "Generalized fuzzy rough sets," Information Sciences, vol. 151, pp. 263-282, 2003.

[17] S. K. Pal, "Soft data mining computational theory of perceptions and rough-fuzzy approach," Information Sciences, vol. 163, no. 13, pp. 5-12, 2004.

[18] S. K. Pal and P. Mitra, "Case generation using rough sets with fuzzy representations," IEEE Transactions on Knowledge and Data Engineering, vol. 16, no. 3, pp. 292-300, 2004.

[19] N. N. Morsi and M. M. Yakout, "Axiomatics for fuzzy rough sets," Fuzzy Sets and Systems, vol. 100, no. 1-3, pp. 327-342, 1998.

[20] D. S. Yeung, D. Chen, E. C. C. Tsang, J. W. T. Lee, and X. Wang, "On the generalization of fuzzy rough sets," IEEE Transactions on Fuzzy Systems, vol. 13, pp. 343-361, 2005.

[21] J. Kortelainen, "On relationship between modified sets, topological spaces and rough sets," Fuzzy Sets and Systems, vol. 61, no. 1, pp. 91-95, 1994.

[22] E. F. Lashin, A. M. Kozae, A. A. Abo Khadra, and T. Medhat, "Rough set theory for topological spaces," International Journal of Approximate Reasoning, vol. 40, no. 1-2, pp. 35-43, 2005.

[23] Z. Li, T. Xie, and Q. Li, "Topological structure for generalized rough sets," Computers \& Mathematics with Applications, vol. 63, no. 6, pp. 1066-1071, 2012.

[24] Z. Pei, D. Pei, and L. Zheng, "Topology versus generalized rough sets," International Journal of Approximate Reasoning, vol. 52, no. 2, pp. 231-239, 2011.

[25] Q. Wu, T. Wang, Y. Huang, and J. Li, “Topology theory on rough sets," IEEE Transactions on Systems, Man, and Cybernetics B, vol. 38, no. 1, pp. 68-77, 2008.

[26] L. Yang and L. Xu, "Topological properties of generalized approximation spaces," Information Sciences, vol. 181, no. 17, pp. 3570-3580, 2011.

[27] Y. Y. Yao, "Two views of the theory of rough sets in finite universes,' International Journal of Approximate Reasoning, vol. 15, no. 4, pp. 291-317, 1996.

[28] K. Qin and Z. Pei, "On the topological properties of fuzzy rough sets," Fuzzy Sets and Systems, vol. 151, no. 3, pp. 601-613, 2005.

[29] J. Hao and Q. Li, “The relationship between $L$-fuzzy rough set and L-topology," Fuzzy Sets and Systems, vol. 178, pp. 74-83, 2011.

[30] L. Zhou, W.-Z. Wu, and W.-X. Zhang, "On intuitionistic fuzzy rough sets and their topological structures," International Journal of General Systems, vol. 38, no. 6, pp. 589-616, 2009. 
[31] W. Wu and L. Zhou, "On intuitionistic fuzzy topologies based on intuitionistic fuzzy reflexive and transitive relations," Soft Computing, vol. 15, pp. 1183-1194, 2011.

[32] M. Kryszkiewicz, "Rough set approach to incomplete information systems," Information Sciences, vol. 112, no. 1-4, pp. 39-49, 1998.

[33] M. Kryszkiewicz, "Rules in incomplete information systems," Information Sciences, vol. 113, no. 3-4, pp. 271-292, 1999.

[34] A. S. Salama, "Topological solution of missing attribute values problem in incomplete information tables," Information Sciences, vol. 180, no. 5, pp. 631-639, 2010.

[35] L. A. Zadeh, "Fuzzy sets," Information and Computation, vol. 8, pp. 338-353, 1965.

[36] Y. Liu and M. Luo, Fuzzy Topology, World Scientific Publishing, Singapore, 1998.

[37] R. Lowen, "Fuzzy topological spaces and fuzzy compactness," Journal of Mathematical Analysis and Applications, vol. 56, no. 3, pp. 621-633, 1976.

[38] C. L. Chang, "Fuzzy topological spaces," Journal of Mathematical Analysis and Applications, vol. 24, pp. 182-190, 1968.

[39] H. Lai and D. Zhang, "Fuzzy preorder and fuzzy topology," Fuzzy Sets and Systems, vol. 157, no. 14, pp. 1865-1885, 2006.

[40] Y. Y. Yao, "Relational interpretations of neighborhood operators and rough set approximation operators," Information Sciences, vol. 111, no. 1-4, pp. 239-259, 1998.

[41] J. Mi, W. Wu, and W. Zhang, "Constructive and axiomatic approaches for the study of the theory of rough sets," Pattern Recognition Artificial Intelligence, vol. 15, pp. 280-284, 2002. 


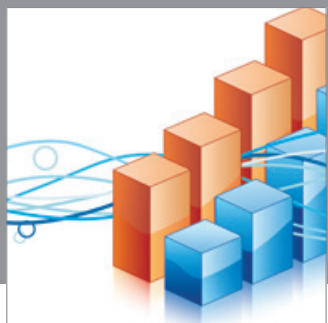

Advances in

Operations Research

mansans

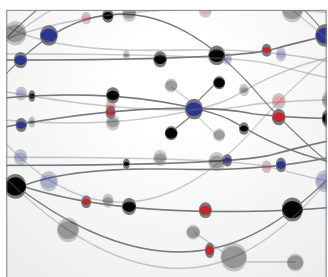

The Scientific World Journal
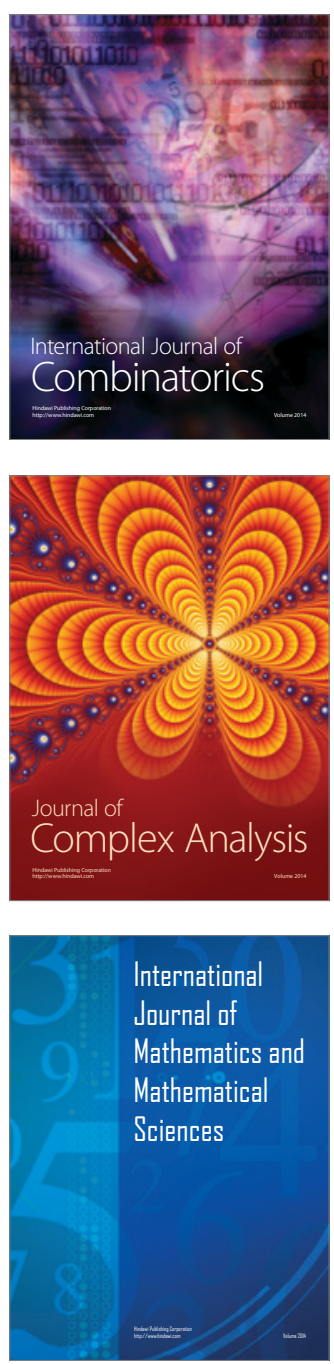
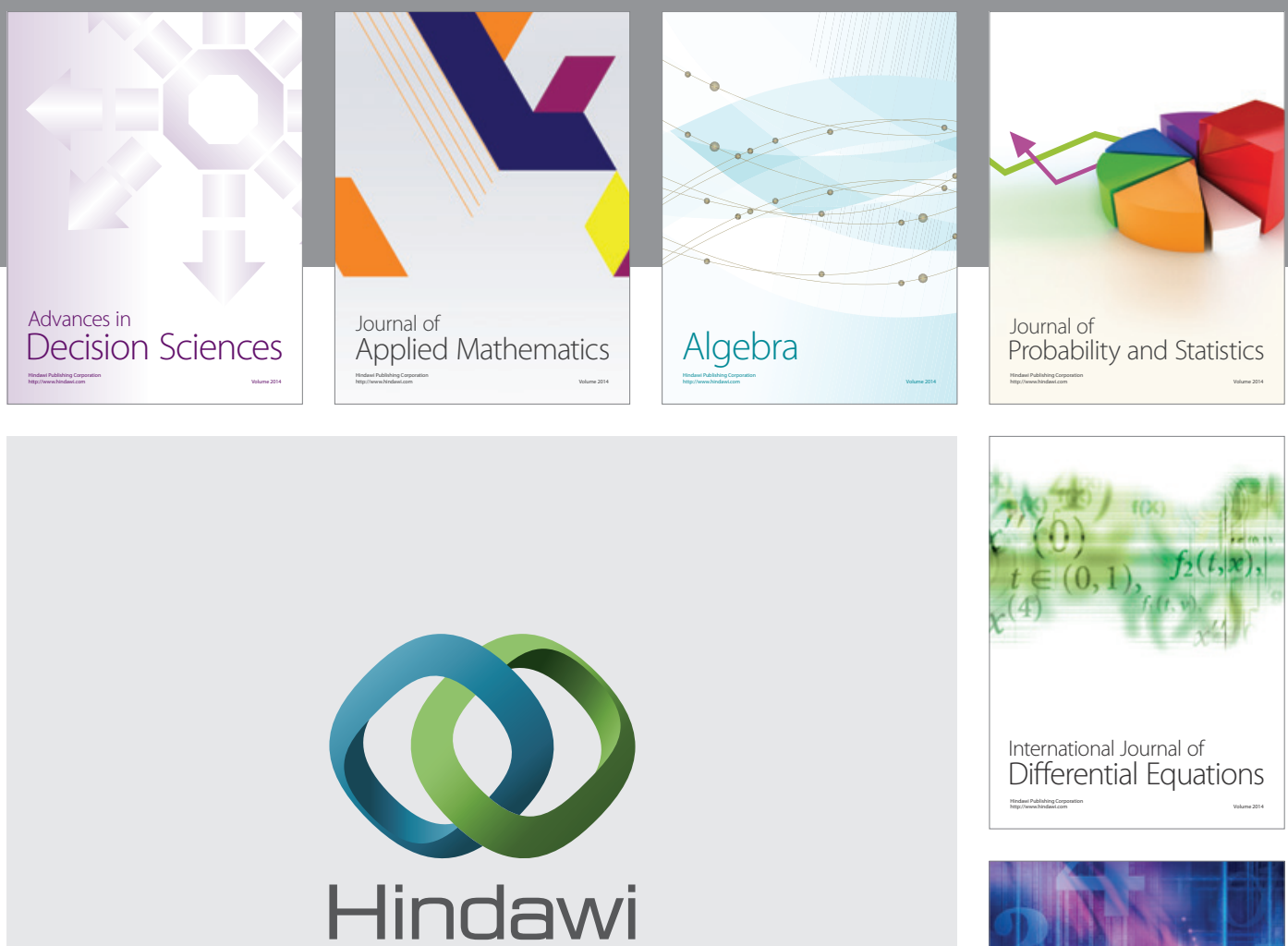

Submit your manuscripts at http://www.hindawi.com
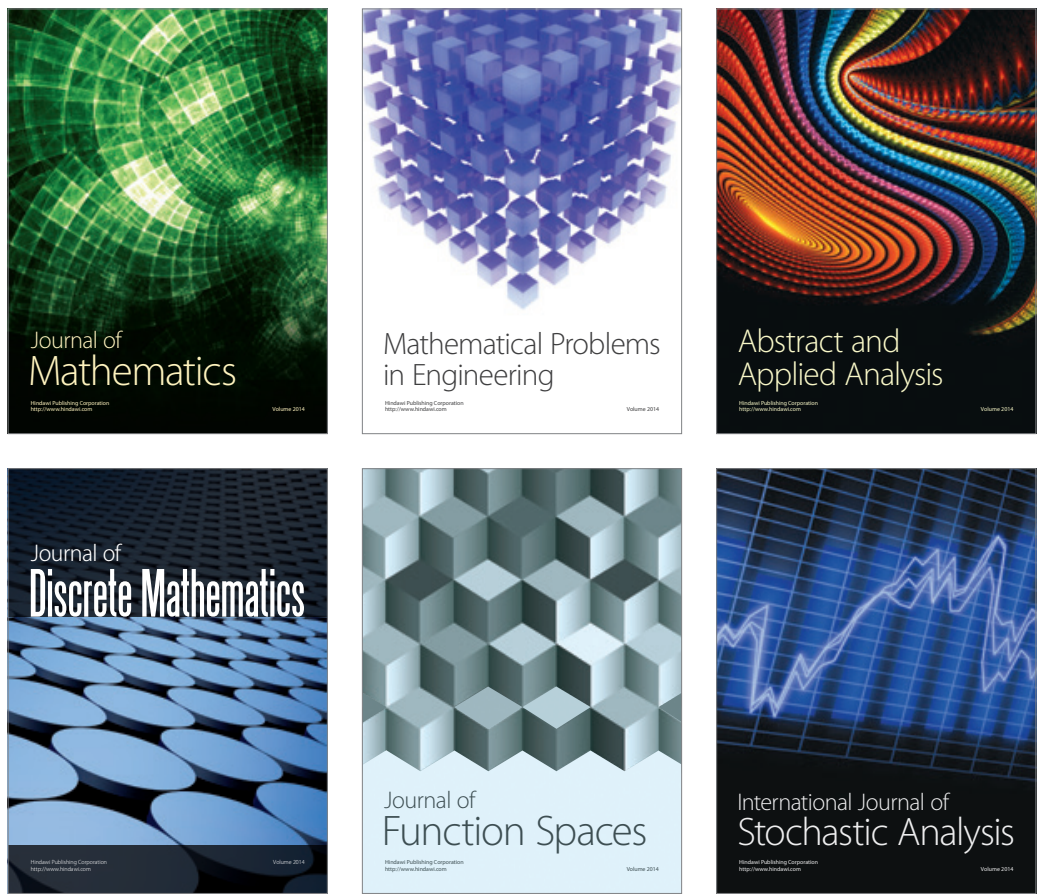

Journal of

Function Spaces

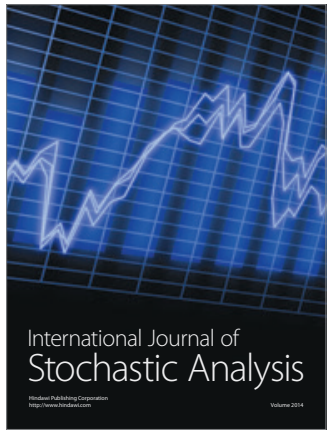

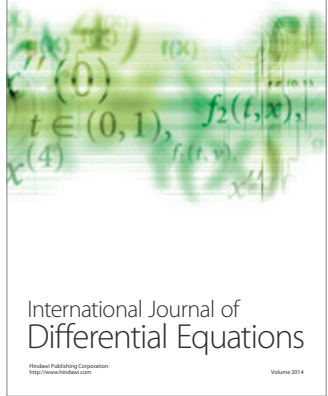
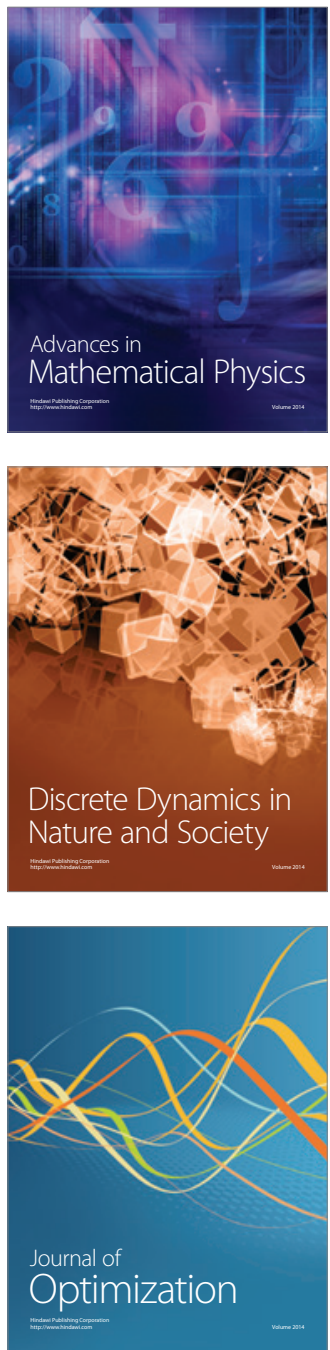